Pacific Journal of Mathematics

TIME-HARMONIC SOLUTIONS OF SOME DISSIPATIVE
PROBLEMS FOR MAXWELLS EQUATIONS IN A
THREE-DIMENSIONAL HALF SPACE 


\section{TIME-HARMONIC SOLUTIONS OF SOME DISSIPATIVE PROBLEMS FOR MAXWELL'S EQUATIONS IN A THREE-DIMENSIONAL HALF SPACE}

\section{J. R. SCHULENBERGER}

In constructing solutions of steady-state wave propagation problems, a common procedure is to assume that the frequency has a small imaginary part and, with an eventual solution in hand, to let this imaginary part go to zero - the principle of limiting absorption. There are three basic problems involved here. The first is to establish the principle of limiting absorption itself, i.e., to show in a rigorous manner that a steady-state solution can actually be obtained in this fashion. The second problem is to find a class of functions in which the solution so constructed is unique (a "radiation condition"). While in problems in exterior domains or with bounded perturbations of the coefficients uniqueness classes are essentially dictated by the asymptotic behavior at large spatial distances of the free-space Green functions, in the problems with infinite boundary considered below it is not immediately clear how to specify uniqueness classes. For example, must the asymptotic behavior at large distances of eventual surface-wave components of the solution be included in the conditions designating such classes? Finally, since, strictly speaking, steadystate solutions are physically meaningless (they fail to have finite energy), a third problem is to determine in what sense they are approximations for large times to the original time-dependent solutions-the principle of limiting amplitude. In this paper we study these questions for the steady-state versions of the dissipative problems for Maxwell's equations considered previously. While these problems are particular examples, the results obtained do provide a guide and generalize to other problems. For example, although the equations of elasticity are much more difficult to deal with, the steady-state Rayleigh surface wave of elasticity theory has basically the same properties as the surface wave in the selfadjoint Leontovich case considered below. As far as we know, questions of uniqueness for steady-state wave propagation problems in domains with infinite boundary have not been considered previously, although Eidus has recently established the principle of limiting absorption for some such domains. Unfortunately, the abstract approach used there apparently does not provide enough information to make it possible to define uniqueness classes for the solutions so constructed.

0. Introduction. To formulate the problem, let $E=\operatorname{diag}\left(\varepsilon I_{3}, \mu I_{3}\right)$ be the diagonal $6 \times 6$ matrix with diagonal elements $\varepsilon, \mu$ which are the electromagnetic constants of a homogeneous, isotropic, loss-free 
medium filling the halfspace $R_{+}^{3}=\left\{x \in R^{3}: x_{3}>0\right\}$. Let $A(D)$, $D_{j}=-i \partial_{J}, j=1,2,3$, be the $6 \times 6$ matrix operator

$$
A(D)=\sum_{j=1}^{3} A_{j} D_{j}=\left[\begin{array}{cc}
0 & i \operatorname{rot} \\
-i \operatorname{rot} & 0
\end{array}\right], \quad \operatorname{rot}=\left[\begin{array}{ccc}
0 & -\partial_{3} & \partial_{2} \\
\partial_{3} & 0 & -\partial_{1} \\
-\partial_{2} & \partial_{1} & 0
\end{array}\right] .
$$

With $\Lambda(D)=E^{-1} A(D)$ we now formulate the problem with the Leontovich boundary condition of finding time-harmonic solutions of

$$
\begin{aligned}
& i \partial_{t} u(x, t)-\Lambda(D) u(x, t)=-f(x) \exp (-i \nu t), \quad x \in R_{+}^{3}, \\
& u_{1}\left(x^{\prime}, 0, t\right)+\alpha u_{5}\left(x^{\prime}, 0, t\right)=u_{2}\left(x^{\prime}, 0, t\right)-\alpha u_{4}\left(x^{\prime}, 0, t\right)=0, \\
& x^{\prime}=\left(x_{1}, x_{2}\right) \in R^{2}, \quad t>0, \quad \alpha \in C, \quad \operatorname{Re} \alpha \geq 0, \quad \nu \neq 0, \\
& f \in C_{0}^{\infty}\left(R_{+}^{3}, C^{6}\right),
\end{aligned}
$$

$$
\begin{aligned}
& i \partial_{t} u^{\prime}+\Lambda(D) u^{\prime}(x, t)=f(x) \exp (i \nu t), \quad x \in R_{+}^{3}, \\
& u_{1}^{\prime}\left(x^{\prime}, 0, t\right)-\bar{\alpha} u_{5}^{\prime}\left(x^{\prime}, 0, t\right)=u_{2}^{\prime}\left(x^{\prime}, 0, \bar{t}\right)+\bar{\alpha} u_{4}^{\prime}\left(x^{\prime}, 0, t\right)=0 .
\end{aligned}
$$

If $v(x), v^{\prime}(x)$ are solutions of

$$
[\Lambda(D)-\nu I] v(x)=f(x), \quad[\Lambda(D)-\nu I] v^{\prime}(x)=f(x)
$$

satisfying the respective boundary conditions indicated in $(0.2),(0.3)$, then the functions

$$
u(x, t)=\exp (-i \nu t) v(x), \quad u^{\prime}(x, t)=\exp (i \nu t) v^{\prime}(x)
$$

will be the desired time-harmonic solutions.

After recalling the required information from [1] in $\S 1$, we justify the principle of limiting absorption and construct such solutions in $\S 2$; we then determine their asymptotic behavior for $|x| \rightarrow \infty$ in $R_{+}^{3}$. In $\S 3$ we prove uniqueness of these solutions in appropriate "radiation" classes. It turns out that in the nonselfadjoint case $\left(\alpha_{1}>0\right)$ we are able to prove uniqueness only for solutions $v(x), v^{\prime}(x)$ for which $\exp (-i \nu t) v(x), \exp (i \nu t) v^{\prime}(x)$ behave for large $|x|$ like outgoing hemispherical waves in $R_{+}^{3}$. We show via the limiting-amplitude principle that these are precisely the physically interesting solutions. Further, it is found that the asymptotic behavior of the surface-wave component of the solution must be included in the designation of a uniqueness class only in the selfadjoint Leontovich case $\alpha=i \alpha_{2}, \alpha_{2} \neq 0$, i.e., when the surface modes from which the solution is formed have real 
frequencies. Designation of uniqueness classes in this case is more difficult (and more interesting), since asymptotically spatial waves are hemispherical waves decaying like $|x|^{-1}$ while surface waves are cylindrical waves decaying only like $|x|^{-1 / 2}$ along the boundary $\left\{x_{3}=0\right\}$.

As mentioned above, versions of the limiting-absorption principle have been widely used. We note that for hyperbolic systems with elliptic spatial part the principle in a half space was established in a rigorous manner for selfadjoint problems by Matsumura [4]. For the same such systems the principle of limiting amplitude was established by Wakabayashi [10].

1. Background. In this section we recall the information from [2] needed below and record the principle of stationary phase in the form required in the next section. Below ${ }^{t} M$ denotes the transpose of a matrix $M,{ }^{\bar{t}} M$ denotes the conjugate transpose, and the adjoint of a matrix or operator is denoted by $M^{*}$.

With respect to the $E$ inner product (see (0.2)) in $C^{6},(\alpha, \beta)={ }^{\bar{t}} \alpha E \beta$, the symbol $\Lambda(\eta), \eta \in R^{3} \backslash\{0\}$ of the operator $\Lambda(D)$ of $(0.2)$,

(1.1) $\Lambda(\eta)=\left[\begin{array}{cc}0 & -\varepsilon^{-1} \eta \wedge \\ \mu^{-1} \eta \wedge & 0\end{array}\right], \quad \eta \wedge=\left[\begin{array}{ccc}0 & -\eta_{3} & \eta_{2} \\ \eta_{3} & 0 & -\eta_{1} \\ -\eta_{2} & \eta_{1} & 0\end{array}\right]$

is selfadjoint with distinct eigenvalues $\lambda_{j}(\eta)=j c|\eta|, j=0, \pm 1, c^{2}=$ $(\varepsilon \mu)^{-1}$, each of multiplicity two. The resolution of the identity for $\Lambda(\eta)$ is

$$
\begin{aligned}
I & =P_{0}(\omega)+P_{1}(\omega)+P_{-1}(\omega) \\
\Lambda(\eta) & =\lambda_{1}(\eta) P_{1}(\omega)+\lambda_{-1}(\eta) P_{-1}(\omega), \quad \omega=\eta /|\eta|
\end{aligned}
$$

where the $P_{k}(\omega)$ are mutually orthogonal orthoprojectors with respect to the $E$ inner product:

$$
\begin{aligned}
{ }^{\bar{t}}\left(E P_{k}\right) & =E P_{k}, \quad \delta_{j k} P_{k}=P_{j} P_{k}, \quad k, j=0, \pm 1, \\
P_{0}(\omega) & =\left[\begin{array}{cc}
{ }^{t} \omega \omega & 0 \\
0 & { }^{t} \omega \omega
\end{array}\right], \\
P_{j}(\omega) & =2^{-1}\left[\begin{array}{cc}
-\omega \wedge \omega \wedge & -j \mu c \omega \wedge \\
j \varepsilon c \omega \wedge & -\omega \wedge \omega \wedge
\end{array}\right] \\
\omega \wedge \omega \wedge & =(\omega \wedge)^{2}, \quad \omega=\eta /|\eta| \in S^{2}, \quad j= \pm 1 .
\end{aligned}
$$


In the space $\mathscr{X}$ of functions $f, g \in L_{2}\left(R_{+}^{3}, C^{6}\right)$ with linear product

$$
(f, g)=\int_{R_{+}^{3}}^{\bar{t}} f E G
$$

the operators $\Lambda, \Lambda^{*}$ defined as the closure of $\Lambda(D)$ on $\left\{f \in C_{0}^{\infty}\left(\bar{R}_{+}^{3}, C^{6}\right)\right.$ : $\left.B f\left(x^{\prime}, 0\right)=0, x^{\prime}=\left(x_{1}, x_{2}\right)\right\},\left\{f \in C_{0}^{\infty}\left(\bar{R}_{+}^{3}, C^{6}\right): B^{\prime} f\left(x^{\prime}, 0\right)=0\right\}$, where

$$
\begin{aligned}
B f\left(x^{\prime}, 0\right) & ={ }^{t}\left(f_{1}+\alpha f_{5}, f_{2}-\alpha f_{4}\right)\left(x^{\prime}, 0^{+}\right), \\
B^{\prime} f\left(x^{\prime}, 0\right) & ={ }^{t}\left(f_{1}-\bar{\alpha} f_{5}, f_{2}+\bar{\alpha} f_{4}\left(x^{\prime}, 0^{+}\right),\right.
\end{aligned}
$$

and $\alpha_{1}+i \alpha_{2}, \alpha_{1}>0, \alpha_{2} \neq 0$. The first case is that of the classical boundary condition [8], while the second is the case considered in [3]. In both these cases $\Lambda$ is selfadjoint, and the spectrum of $\Lambda, \sigma(\Lambda)$ consists of the entire real line $R$. In the third case studied in [2] (which includes the second case) the spectrum of $\Lambda$ consists of three parts:

$$
\begin{aligned}
\bar{\sigma}\left(\Lambda^{*}\right) & =\sigma(\Lambda)=R \cup\{m\} \cup\{e\} \\
\{m\} & =\{m(\lambda)=-p \lambda / \varepsilon, \lambda \in(0, \infty)\} \\
\{e\} & =\{e(\lambda)=c q \lambda, \lambda \in(0, \infty)\}, \\
p & =p_{1}+i p_{2}, \quad p_{2} \geq 0, \quad \alpha_{2} p_{1}>0, \quad p^{2}=1 /\left(\mu \varepsilon^{-1}-\alpha^{2}\right), \\
q & =q_{1}+i q_{2}, \quad q_{2} \leq 0, \quad \alpha_{2} q_{1}>0, \quad q^{2}=-\alpha^{2} p^{2} .
\end{aligned}
$$

In the general case, the spectrum thus consists of $R$ plus two lines in the lower half plane issuing from the origin to the left and right of the negative imaginary axis.

To points of $R$ there correspond generalized eigenfunctions of $\Lambda(D)$, $\Lambda^{*}(D)$ satisfying $\Lambda(D) \Psi_{j}(x, \eta)_{ \pm}=\lambda_{J}(\eta) \Psi_{j}(x, \eta)_{ \pm}, \Lambda^{*}(D) \Psi_{j}^{\prime}(x, \eta)_{ \pm}=$ $\lambda_{j}(\eta) \Psi_{j}^{\prime}(x, \eta)_{ \pm}, j= \pm 1$, and the boundary conditions $(1.4),\left(1.4^{\prime}\right)$ given by

$$
\begin{aligned}
& \Psi_{j}(x, \eta)_{ \pm} \\
& \quad=(2 \pi)^{-3 / 2} \chi_{\mp j}(\rho) e^{i x^{\prime} \xi}\left[e^{i \rho x_{3}} P_{j}(\omega)-e^{-i \rho x_{3}} C_{j}(\omega) P_{j}(\omega)\right] E^{-1} \\
& \Psi_{j}^{\prime}(x, \eta)_{ \pm} \\
& \quad=(2 \pi)^{-3 / 2} \chi_{\mp j}(\rho) e^{i x^{\prime} \xi}\left[e^{i \rho x_{3}} P_{j}(\omega)-e^{-i \rho x_{3}} C_{j}^{\prime}(\omega) P_{j}(\omega)\right] E^{-1}
\end{aligned}
$$

where we have written $\eta=(\xi, \rho) \in R^{3}, \chi_{\mp j}$ are the characteristic functions of the half spaces $R_{\mp j}^{3}, j= \pm 1$ (e.g., $R_{\mp j}^{3}=R_{ \pm}$for $j=-1$ ), 
and

$$
\begin{aligned}
& C(\omega)=\Delta(\omega)^{-1}\left[\varepsilon c \omega_{3}\left(\alpha^{2}-\mu \varepsilon^{-1}\right) Q+\alpha c\left(\omega^{\prime}\right)\right], \\
& C^{\prime}(\omega)=\Delta^{\prime}(\omega)^{-1}\left[\varepsilon c \omega_{3}\left(\mu \varepsilon^{-1}-\bar{\alpha}^{2}\right) Q+\bar{\alpha} c\left(\omega^{\prime}\right)\right] \text {, } \\
& \Delta(\omega)=\left(\mu c-\alpha \omega_{3}\right)\left(\alpha \varepsilon c-\omega_{3}\right) \text {, } \\
& \Delta^{\prime}(\omega)=\left(\mu c+\bar{\alpha} \omega_{3}\right)\left(\bar{\alpha} \varepsilon c+\omega_{3}\right) \text {, } \\
& Q=\operatorname{diag}[1,1,-1,-1,-1,1] \text {, } \\
& c\left(\omega^{\prime}\right)=\left[\begin{array}{cc}
0 & \tilde{c}\left(\omega^{\prime}\right) \\
\tilde{c}\left(\omega^{\prime}\right) & 0
\end{array}\right] \text {, } \\
& \tilde{c}\left(\omega^{\prime}\right)=\left[\begin{array}{ccc}
\omega_{2}^{2}-\omega_{1}^{2} & -2 \omega_{1} \omega_{2} & 0 \\
-2 \omega_{1} \omega_{2} & \omega_{2}^{2}-\omega_{1}^{2} & 0 \\
0 & 0 & \left|\omega^{\prime}\right|^{2}
\end{array}\right], \\
& C_{j}(\omega)=C\left(\omega^{\prime}, j \omega_{3}\right), \quad C_{j}^{\prime}(\omega)=C^{\prime}\left(\omega^{\prime}, j \omega_{3}\right) \text {. }
\end{aligned}
$$

We observe that

$$
\begin{aligned}
{ }^{\bar{t}} C_{j}(\omega) & =C_{j}^{\prime}(\tilde{\omega}), \quad C_{j}(\omega) C_{j}(\tilde{\omega})=C_{j}(\tilde{\omega}) C_{j}(\omega)=I, \\
\tilde{\omega} & =\left(\omega^{\prime},-\omega_{3}\right), \\
C_{j}(\omega) P_{j}(\omega) & =P_{j}(\tilde{\omega}) C_{j}(\omega), \\
C_{j}^{\prime}(\omega) P_{j}(\omega) & =P_{j}(\tilde{\omega}) C_{j}^{\prime}(\omega), \quad j= \pm 1 .
\end{aligned}
$$

To points of $\{e\}$ and $\{m\}$ there correspond generalized eigenfunctions $\Sigma_{S}(x, \xi), \Sigma_{S}^{\prime}(x, \xi)$ satisfying $\Lambda(D) \Sigma_{S}(x, \xi)=s(\xi) \Sigma_{S}(x, \xi)$, $\Lambda^{*}(D) \Sigma_{S}^{\prime}(x, \xi)=\bar{s}(\xi) \Sigma_{S}^{\prime}(x, \xi), S=E, M, s=e, m, e(\xi)=\varepsilon q|\xi|, m=$ $-p|\xi| / \varepsilon$, and the boundary conditions $(1.4),\left(1.4^{\prime}\right)$ given by

$$
\begin{aligned}
\Sigma_{E}(x, \xi) & =(2 \pi)^{-1} \mu \beta_{E}|\xi|^{-7 / 2} \exp \left[i x^{\prime} \xi+i \tau_{e}(\xi) x_{3}\right] \mathscr{E}^{t} \mathscr{L}^{\prime}, \\
\Sigma_{M}(x, \xi) & =(2 \pi)^{-1} \varepsilon \beta_{M}|\xi|^{-7 / 2} \exp \left[i x^{\prime} \xi+i \tau_{m}(\xi) x_{3}\right] \mathscr{M}^{t} \mathscr{M}^{\prime}, \\
\Sigma_{E}^{\prime}(x, \xi) & =(2 \pi)^{-1} \mu \bar{\beta}_{E}|\xi|^{-7 / 2} \exp \left[i x^{\prime} \xi-i \bar{\tau}_{e}(\xi) x_{3}\right] \overline{\mathscr{E}}^{t} \overline{\mathscr{E}}, \\
\Sigma_{M}^{\prime}(x, \xi) & =(2 \pi)^{-1} \mu \bar{\beta}_{M}|\xi|^{-7 / 2} \exp \left[i x^{\prime} \xi-i \bar{\tau}_{m}(\xi) x_{3}\right] \overline{\mathscr{M}}^{t} \overline{\mathscr{M}}, \\
\mathscr{E}(\xi) & ={ }^{t}\left(\xi_{2} e(\xi),-\xi_{1} e(\xi), 0,-\alpha^{-1} \xi_{1} e(\xi),-\alpha^{-1} \xi_{2} e(\xi),-\mu^{-1}|\xi|^{2}\right), \\
\mathscr{M}(\xi) & ={ }^{t}\left(\alpha \xi_{1} m(\xi), \alpha \xi_{2} m(\xi), \varepsilon^{-1}|\xi|^{2}, \xi_{2} m(\xi),-\xi_{1} m(\xi), 0\right), \\
\mathscr{E}^{\prime}(\xi) & ={ }^{t}\left(\xi_{2} e(\xi),-\xi_{1} e(\xi), 0, \alpha^{-1} \xi_{1} e(\xi), \alpha^{-1} \xi_{2} e(\xi),-\mu^{-1}|\xi|^{2}\right), \\
\mathscr{M}^{\prime}(\xi) & ={ }^{t}\left(\alpha \xi_{1} m(\xi), \alpha \xi_{2} m(\xi),-\varepsilon^{-1}|\xi|^{2},-\varepsilon_{2} m(\xi), \xi_{1} m(\xi), 0\right), \\
\beta_{E} & =(i q / 2 \alpha c \varepsilon)^{1 / 2}, \quad \beta_{M}=(-i p \alpha / 2)^{1 / 2}, \quad \operatorname{Im}(\cdot)^{1 / 2} \geq 0, \\
\tau_{m}(\xi) & =\alpha p|\xi|, \quad \tau_{e}(\xi)=-\alpha^{-1} \mu c q|\xi|, \quad \operatorname{Im} \tau_{m, e}(\xi) \geq 0 .
\end{aligned}
$$


Denoting by $\mathscr{K}_{n}, n=2,3$, the space of functions $f, g \in L_{2}\left(R^{n}, C^{6}\right)$ with the inner product $(f, g)_{\mathscr{\varkappa}_{n}}=\langle f, E g\rangle$, the functions $\Psi_{j}, \Psi_{j}^{\prime}, \Sigma_{S}$, $\Sigma_{S}^{\prime}, S=E, M, j= \pm 1$, define bounded operators from $\mathscr{X}$ to $\mathscr{K}_{n}$ obtained by affixing the appropriate subscript and eventual prime to

$$
\begin{aligned}
& \Psi f(\eta)=\int_{R_{+}^{3}} \bar{t} \Psi(x, \eta) E f(x) d x \\
& \Sigma f(\xi)=\int_{R_{+}^{3}}{ }^{\bar{t}} \Sigma(x, \xi) E f(x) d x .
\end{aligned}
$$

Their adjoints are

$$
\begin{aligned}
& \Psi^{*} g(x)=\int_{R^{3}} \Psi(x, \eta) E g(\eta) d \eta \\
& \Sigma^{*} h(x)=\int_{R^{2}} \Sigma(x, \xi) E h(\xi) d \xi
\end{aligned}
$$

The operators $\Pi_{j}=\Psi_{j}^{*} \Psi_{j}^{\prime}, \Pi_{S}=\Sigma_{S}^{*} \Sigma_{S}^{\prime}, j= \pm 1, S=E, M$, and their adjoints are bounded projections in $\mathscr{X}$ which are "orthogonal" in the sense that $0=\Pi_{j} \Pi_{j^{\prime}}=\Pi_{j} \Pi_{S}=\Pi_{S} \Pi_{j}=\Pi_{E} \Pi_{M}=\Pi_{M} \Pi_{S}$, $j \neq j^{\prime}=0, \pm 1, S=E, M$. Here $\Pi_{0}$ is the orthogonal projection onto the null space of $\Lambda, \mathscr{N}(\Lambda)=\mathscr{N}\left(\Lambda^{*}\right)$, defined by (see formula (A.9), p. 174 of [2])

(1.12) $\Phi_{2} \Pi_{0} f\left(\xi, x_{3}\right)$

$$
\begin{aligned}
= & (2 \pi)^{-1 / 2} \int_{R} \exp \left(i \rho x_{3}\right) P_{0}(\xi, \rho) \Phi_{3} \chi_{+} f(\xi, \rho) d \rho \\
& -2^{-1}(2 \pi)^{1 / 2} \exp \left(-|\xi| x_{3}\right) \widetilde{P}_{0}^{r}(\xi,-i|\xi|) \Phi_{3} \chi_{+} f(\xi,-i|\xi|)
\end{aligned}
$$

where $\Phi_{n}, n=2,3$, is the Fourier transform

$$
\Phi_{n} f(\eta)=(2 \pi)^{-n / 2} \int_{R_{n}} \exp (-i x \eta) f(x) d x
$$

with inverse $\Phi_{n}^{*} f(x)=\Phi_{n} f(-x), \chi_{+}$is the characteristic function of the half space $R_{+}^{3}, P_{0}(\xi, \rho)$ is given in (1.3), and

$$
P_{o}^{r}(\xi,-i|\xi|)=\left[\begin{array}{cc}
{ }^{t} d(\xi) \bar{d}(\xi) & 0 \\
0 & { }^{t} d(\xi) \bar{d}(\xi)
\end{array}\right], \begin{aligned}
& \\
& \\
&
\end{aligned}
$$


The semigroups $S(t)=\exp (-i \Lambda t), S^{*}(t)=\exp \left(i \Lambda^{*} t\right)$ generated by $\Lambda$, $\Lambda^{*}$ have the representations

$$
\begin{gathered}
\text { (1.14) } \begin{array}{c}
S(t) f=\Pi_{0} f+\Psi_{1}^{*} \exp (-i c|\cdot| t) \Psi_{1}^{\prime} f+\Psi_{-1}^{*} \exp (i c|\cdot| t) \Psi_{-1}^{\prime} f \\
+\Sigma_{E}^{*} \exp (-i e(\cdot) t) \Sigma_{E}^{\prime} f+\Sigma_{M}^{*} \exp (-i m(\cdot) t) \Sigma_{M}^{\prime} f \\
S^{*}(t) f=\Pi_{0} f+\left(\Psi_{1}^{\prime}\right)^{*} \exp (i c|\cdot| t) \Psi_{1} f+\left(\Psi_{-1}^{\prime}\right)^{*} \exp (-i c|\cdot| t) \Psi_{-1} f \\
+\left(\Sigma_{E}^{\prime}\right)^{*} \exp (i \bar{e}(\cdot) t) \Sigma_{E} f+\left(\Sigma_{M}^{\prime}\right)^{*} \exp (i \bar{m}(\cdot) t) \Sigma_{M} f
\end{array}
\end{gathered}
$$

which for $t=0$ are the Parseval identities for $\Lambda, \Lambda^{*}$. The last two terms on the right in (1.14) do not occur in the selfadjoint case $\alpha=0$.

Suppose now that $R(s)$ is a smooth, matrix-valued function on the unit sphere $S^{2} \ni s, \omega, x=|x| \omega \in R_{+}^{3}, y \in R_{+}^{3}, r>0$, and $\psi(r, y, s)$ is a smooth function. The principle of stationary phase in the form we require in the next section (see, e.g., [4], [9]) states that as $|x| \rightarrow$ $\infty$, uniformly with respect to $r$ in bounded intervals of $R_{+}$and $y$ in compact sets

$$
\begin{aligned}
& \int_{S^{2}} \exp (i r x s) \psi(r, y, s) R(s) d S \\
& =(2 \pi) \sum_{j= \pm 1}\{\exp (i j r|x|-i j \pi / 2) \psi(r, y, j \omega) \\
& \left.\quad R(j \omega)|r x|^{-1}\right\}+q(r x), \\
& D^{\alpha} q(x)=O\left(|x|^{-2}\right) \text { and smooth. }
\end{aligned}
$$

For $R^{2} \ni x^{\prime}=\left|x^{\prime}\right| \gamma, \gamma=\left(\cos \phi_{x}, \sin \phi_{x}\right)$, and $s=(\cos \phi, \sin \phi) \in S^{1}$ the corresponding statement is

$$
\begin{aligned}
& \int_{S^{1}} \exp \left(i r x^{\prime} s\right) \psi(r, y, s) R(s) d \phi \\
& =(2 \pi)^{1 / 2}\left|r x^{\prime}\right|^{-1 / 2} \\
& \quad \times \sum_{j= \pm 1}\left\{\exp \left(i j r\left|x^{\prime}\right|-i j \pi / 4\right) \psi(r, y, j \gamma) R(j \gamma)\right\}+q\left(r x^{\prime}\right) \\
& \quad D^{\alpha} q\left(x^{\prime}\right)=O\left(\left|x^{\prime}\right|^{-3 / 2}\right) \text { and smooth. }
\end{aligned}
$$

2. The limiting-absorption principle and asymptotics. It follows directly from (1.10)-(1.12), (1.14) that for $\zeta \notin \sigma(\Lambda), f \in C_{0}^{\infty}\left(R_{+}^{3}, C^{6}\right)$

$$
\begin{aligned}
(2.1) v(x, \zeta)= & -\zeta^{-1} \Pi_{0} f(x)+\sum_{j= \pm 1} \Psi_{j}^{*}\left[\lambda_{j}(\cdot)-\zeta\right]^{-1} \Psi_{j}^{\prime} f(x) \\
& +\Sigma_{E}^{*}[e(\cdot)-\zeta]^{-1} \Sigma_{E}^{\prime} f(x)+\Sigma_{M}^{*}[m(\cdot)-\zeta]^{-1} \Sigma_{M}^{\prime} f(x) \\
\equiv & v_{0}(x ; \zeta)+v_{-1}(x ; \zeta) \\
& +v_{1}(x ; \zeta)+v_{E}(x ; \zeta)+v_{M}(x ; \zeta),
\end{aligned}
$$




$$
\begin{aligned}
\left(2.1^{\prime}\right) v^{\prime}(x ; \bar{\zeta})= & -\bar{\zeta}^{-1} \Pi_{0} f(x)+\sum_{j= \pm 1}\left(\Psi_{j}^{\prime}\right)^{*}\left[\lambda_{j}(\eta)-\bar{\zeta}\right]^{-1} \Psi_{j} f(x) \\
& +\left(\Sigma_{E}^{\prime}\right)^{*}[e(\cdot)-\bar{\zeta}]^{-1} \Sigma_{E} f(x) \\
& +\left(\Sigma_{M}^{\prime}\right)^{*}[m(\cdot)-\bar{\zeta}]^{-1} \Sigma_{M} f(x) \\
\equiv & v_{0}(x ; \bar{\zeta})+v_{-1}^{\prime}(x ; \bar{\zeta})+v_{1}^{\prime}(x ; \bar{\zeta})+v_{E}^{\prime}(x ; \bar{\zeta})+v_{M}^{\prime}(x ; \bar{\zeta})
\end{aligned}
$$

are solutions of

$$
\begin{aligned}
& {[\Lambda(D)-\zeta I] v(x ; \zeta)=f(x), \quad B v\left(x^{\prime}, 0 ; \xi\right)=0} \\
& {[\Lambda(D)-\bar{\zeta} I] v^{\prime}(x ; \bar{\zeta})=f(x), \quad B^{\prime} v^{\prime}\left(x^{\prime}, 0 ; \bar{\xi}\right)=0}
\end{aligned}
$$

We now take $\zeta=\nu \pm i \varepsilon, \nu \neq 0, \varepsilon \in\left(0, \varepsilon_{0}\right]$ (with $\varepsilon_{0}$ so small that $\nu-i \varepsilon_{0}$ is not in $\sigma(\Lambda)$ in the case $-i \varepsilon)$ and pass to the limit $\varepsilon \rightarrow 0$ to obtain solutions $v_{ \pm}(x, \nu)=v(x ; \nu \pm i 0), v_{ \pm}^{\prime}(v ; \nu)=v^{\prime}(x ; \nu \pm i 0)$ of

$$
\begin{aligned}
& {[\Lambda(D)-\nu I] v(x)=f(x), \quad x \in R_{+}^{3}, \quad B v\left(x^{\prime}, 0\right)=0} \\
& {[\Lambda(D)-\nu I] v^{\prime}(x)=f(x), \quad x \in R_{+}^{3}, \quad B^{\prime} v^{\prime}\left(x^{\prime}, 0\right)=0}
\end{aligned}
$$

This forms the content of the limiting-absorption principle. We then determine the asymptotic behavior as $|x| \rightarrow \infty$ of each component of the solutions $v_{ \pm}, v_{ \pm}^{\prime}$. This provides the means of specifying uniqueness classes for them.

1. $v_{0}(x ; \nu \pm i 0)=-\nu^{-1} \Pi_{0} f(x)$. We consider $f \in C_{0}^{\infty}\left(R_{+}^{3}, C^{6}\right)$ extended by zero to $R^{3}$. From (1.12)

$$
\begin{aligned}
\Pi_{0} f(x) & =(2 \pi)^{-3 / 2} \Phi_{3}^{*} P_{0} \Phi_{3} f(x)-2^{-1}(2 \pi)^{-1 / 2} R(x), \\
R(x) & =\int_{R_{2}} \exp \left(i x^{\prime} \xi-|\xi| x_{3}\right)|\xi|^{-1} \widetilde{P}_{0}^{r}(\xi,-i|\xi|) \phi(\xi) d \xi, \\
\phi(\xi) & =\int_{0}^{\infty} \exp \left(-|\xi| y_{3}\right) \Phi_{2} f\left(\xi, y_{3}\right) d y_{3} .
\end{aligned}
$$

The first term of $\Pi_{0} f(x)$ is a singular integral operator, so (cf. [6], [7])

$$
(2 \pi)^{-3 / 2} \Phi_{3}^{*} P_{0} \Phi_{3} f(x)=O\left(|x|^{-3}\right), \quad|x| \rightarrow \infty .
$$

For $x_{3}=0$ we obtain by integration by parts

$$
\left|x^{\prime}\right|^{2}\left|R\left(x^{\prime}, 0\right)\right| \leq c(f)<\infty .
$$

Since $R(x)$ is a harmonic function, (2.6) implies that it can be represented in $R^{3}$ by Poisson's formula, but this does not give an estimate which is uniform in $\omega_{3}=x_{3} /|x|$. Proceeding directly from (1.12), 
(1.13), (2.4), we have for the $i$ th column of $R, x_{3}>0, \operatorname{supp} f(x) \subset$ $K \times[a, b], 0<a<b<\infty, K \subset R^{2}$ bounded, $i=1, \ldots, 6$,

$$
\begin{aligned}
\left|x_{3}^{2} R_{i}(x)\right| & \leq c(f) x_{3}^{2} \int_{0}^{\infty} \exp \left[-|\xi|\left(x_{3}+y_{3}\right)\right]|\xi|^{2} d|\xi| d y_{3} \\
& =c(f) x_{3}^{2} \partial_{3}^{2} \int_{a}^{b}\left(x_{3}+y_{3}\right)^{-1} d y_{3} \\
& =c(f)\left[x_{3}^{2} /\left(x_{3}+a\right)^{2}-x_{3}^{2} /\left(x_{3}+b\right)^{2}\right] \leq c(f)<\infty
\end{aligned}
$$

Now

$$
\begin{aligned}
\left|x^{\prime}\right|^{2}|R(x)|= & \int_{R_{2}}\left[\left(-\Delta_{\xi}\right) \exp \left(i x^{\prime} \xi-|\xi| x_{3}\right)\right]|\xi|^{-1} \widetilde{P}_{0}^{r}(\xi,-i|\xi|) \phi(\xi) d \xi \\
& -2 i \sum_{k=1}^{2} x_{k} x_{3} \int_{R^{2}}\left\{\exp \left(i x^{\prime} \xi-|\xi| x_{3}\right) \xi_{k}|\xi|^{-2}\right. \\
& \left.\times \widetilde{P}_{0}^{r}(\xi,-i|\xi|)\right\} \phi(\xi) d \xi \\
& -x_{3} \int_{R^{2}} \exp \left(i x^{\prime} \xi-|\xi| x_{3}\right)|\xi|^{-2} \widetilde{P}_{0}^{r}(\xi,-i|\xi|) \phi(\xi) d \xi \\
& +x_{3}^{2} R(x) \\
\equiv & \sum_{k=1}^{5} r_{j}(x) .
\end{aligned}
$$

From (2.7) $\left|r_{5}(x)\right| \leq c(f)<\infty$. Integration by parts in $r_{1}(x)$ gives $\left|r_{1}(x)\right| \leq c(f)<\infty$. Just as in (2.7)

$$
\begin{aligned}
\left|r_{4}(x)\right| & \leq c(f) x_{3} \int_{a}^{b} \int_{0}^{\infty} \exp \left[-|\xi|\left(x_{3}+y_{3}\right)\right]|\xi| d|\xi| d y_{3} \\
& =c(f)\left[x_{3} /\left(a+x_{3}\right)-x_{3} /\left(b+x_{3}\right)\right]<c(f)<\infty
\end{aligned}
$$

Finally,

$$
\begin{aligned}
r_{2}(x)+r_{3}(x)= & -2 x_{3} \sum_{k=1}^{2} \int_{R^{2}}\left[\partial_{\xi_{k}} \exp \left(i x^{\prime} \xi-|\xi| x_{3}\right)\right] \xi_{k}|\xi|^{-2} \\
& \times \widetilde{P}_{0}^{r}(\xi,-i|\xi|) \phi(\xi) d \xi-2 x_{3}^{2} R(x) \\
= & -2 x_{3} \sum_{k=1}^{2} \int_{R^{2}} \exp \left(i x^{\prime} \xi-|\xi| x_{3}\right) \xi_{k}|\xi|^{-2} \\
& \times \widetilde{P}_{0}^{r}(\xi,-i|\xi|)(\xi,-i|\xi|) \phi(\xi) d \xi \\
& +2 x_{3} \sum_{k=1}^{2} \int_{R^{2}} \exp \left(i x^{\prime} \xi-|\xi| x_{3}\right) \xi_{k}|\xi|^{-2} \\
& \times \partial_{\xi_{k}} \widetilde{P}_{0}^{r}(\xi,-i|\xi|) \phi(\xi) d \xi-2 x_{3}^{2} R(x) .
\end{aligned}
$$


The last term has already been estimated in (2.7); the estimate for the first four terms is essentially the same as for $r_{4}(x)$ above. Thus, from (2.7), (2.7') we have $|x|^{2} R(x) \leq c(f)<\infty$. From (2.5) and similar estimates for $D^{\beta} v_{0}$ we thus have

(2.8) $\nu|x|^{2}\left|D^{\beta} v_{0}(x ; \nu \pm i 0)\right| \leq c(f)<\infty$,

$$
|x| \rightarrow \infty, \quad x_{3} \geq 0, \quad|\beta| \geq 0 .
$$

2. $v_{1}(x ; \nu \pm i 0)$. Suppose first that $\nu>0$. From $(1.6),(1.8),(1.10)$

(2.9) $\quad \Psi_{1}^{\prime} f(\eta)=\chi_{\mp}(\rho) \check{f}_{1}(\eta), \quad \check{f}_{1}(\eta)=P_{1}(\eta)\left[\Phi_{3} f(\eta)-C_{1}(\tilde{\eta}) \Phi_{3} f(\tilde{\eta})\right]$, $C_{1}(\eta) \check{f}_{1}(\eta)=-\check{f}_{1}(\tilde{\eta}), \quad \tilde{\eta}=(\xi,-\rho)$.

Hence, from (1.11), (2.1), (2.9)

(2.10) $D^{\beta} v_{1}(x ; \zeta)=D^{\beta} \Psi_{1}^{*}\left[\lambda_{1}(\eta)-\zeta\right]^{-1} \Psi_{1}^{\prime} f(\eta)$

$$
\begin{gathered}
=(2 \pi)^{-3 / 2} \int_{R^{3}}\left[\exp (i x \eta) \eta^{\beta}-\exp (i x \tilde{\eta}) \tilde{\eta}^{\beta} C_{1}(\eta)\right] \\
\times[c|\eta|-\zeta]^{-1} \chi_{\mp}(\rho) \check{f}_{1}(\eta) d \eta \\
=(2 \pi)^{3 / 2} \int_{R^{3}} \exp (i x \eta) \eta^{\beta}[c|\eta|-\zeta]^{-1} \check{f}_{1}(\eta) d \eta .
\end{gathered}
$$

Suppose now that $\nu \in\left(\nu_{0}-\delta, \nu_{0}+\delta\right), \nu_{0}-\delta>0$, and let $\psi \in C_{0}^{\infty}(R)$ with supp $\psi \subset\left\{r:\left|c r-\nu_{0}\right| \leq 4 \delta\right\} \subset(0, \infty), \psi(r)=1$ for $\left|c r-\nu_{0}\right| \leq 3 \delta$. Define $\chi \in C_{0}^{\infty}\left(R^{3} /\{0\}\right)$ by $\chi(\eta) \equiv \chi(r s)=\psi(r), s \in S^{2}$. Then for $\zeta=\nu \pm i \varepsilon$ with $\varepsilon \in\left(0, \varepsilon_{0}\right]$ from $(2.10)$

(2.11) $\quad D^{\beta} v_{1}(x ; \zeta)=(2 \pi)^{-3 / 2} \int_{R^{3}} \exp (i x \eta) \eta^{\beta}[c|\eta|-\zeta]^{-1} \chi(\eta) \check{f}_{1}(\eta) d \eta$

$$
\begin{aligned}
& +(2 \pi)^{-3 / 2} \int_{R^{3}} \exp (i x \eta) \eta^{\beta}[c|\eta|-\zeta]^{-1} \\
& \times[1-\chi(\eta)] \check{f}_{1}(\eta) d \eta \\
\equiv I(x ; \zeta)+J(x ; \zeta) . &
\end{aligned}
$$

From (2.9), (2.11)

$$
\begin{aligned}
J(x ; \zeta) & =\Phi_{3}^{*} P_{1} \Phi_{3} g(x)-\Phi_{3}^{*} P_{1} C_{1}(\tilde{)}) \Phi_{3} \tilde{g}(x) \\
\Phi_{3} g(\eta) & =\eta^{\beta}[c|\eta|-\zeta]^{-1}[1-\chi(\eta)] \Phi_{3} f(\eta) \\
\Phi_{3} \tilde{g}(\eta) & =\eta^{\beta}[c|\eta|-\zeta]^{-1}[1-\chi(\eta)] \Phi_{3} f(\tilde{\eta})
\end{aligned}
$$


Since $P_{1}(\eta)$ and $P_{1}(\eta) C_{1}(\tilde{\eta})$ are homogeneous of degree zero, $J(x ; \zeta)$ is thus the sum of two singular integral operators. Since $g$ and $\tilde{g}$ are smooth and rapidly decreasing, it now follows easily (cf., e.g., [6], p. 50) that for any $\mu>0$ uniformly with respect to $\nu \in\left[\nu_{0}-\delta, \nu_{0}+\delta\right]$, $\varepsilon \in\left(0, \varepsilon_{0}\right]$

$$
|J(x ; \nu \pm i 0)| \leq c(f)|x|^{-3+\mu}
$$

From (2.9), (2.11) with $s=\eta /|\eta|$

(2.13) $I(x ; \zeta)=(2 \pi)^{-3} \int_{R^{3}} \int_{R_{+}^{3}} \exp (i x \eta) \eta^{\beta} \chi(\eta)[c|\eta|-\zeta]^{-1} P_{1}(s)$

$$
\begin{aligned}
& \times\left[\exp (-i y \eta)-C_{1}(\tilde{s}) \exp (-i y \tilde{\eta})\right] f(y) d y d \eta \\
= & (2 \pi)^{-3} \int_{R_{+}^{3}} \int_{R^{3}} \chi(\eta) \eta^{\beta}[c|\eta|-\zeta]^{-1} P_{1}(s) \\
& \times\left\{\exp [i(x-y) \eta]-C_{1}(\tilde{s}) \exp [i(x-y) \tilde{\eta}]\right\} f(y) d \eta d y \\
\equiv & (2 \pi)^{-3} \int_{R_{+}^{3}} D(x, y ; \zeta) f(y) d y,
\end{aligned}
$$

$$
\begin{aligned}
D(x, y ; \nu \pm i \varepsilon)=\int_{0}^{\infty}[ & c r-(\nu \pm i \varepsilon)]^{-1} r^{2+\beta} \psi(r) d r \int_{S^{2}} P_{1}(s) s^{\beta} \\
& \times\left\{\exp [\operatorname{ir}(x-y) s]-C_{1}(\tilde{s}) \exp [\operatorname{ir}(x-y) \tilde{s}]\right\} d S .
\end{aligned}
$$

Replacing the interval $\left|c r-\nu_{0}\right| \leq 2 \delta$ by a semicircle of radius $2 \delta<\varepsilon_{0}$ in the lower $(\nu+i \varepsilon)$ or upper $(\nu-i \varepsilon)$ half plane and extending $\psi$ to this half disk by 1 , it follows from (2.14) that $I(x ; \nu \pm i 0)$ exists and is continuous in $(x, \nu), \nu \in\left[\nu_{0}-\delta, \nu_{0}+\delta(\eta)\right]$. Hence, from (2.12) $D^{\beta} v_{1}(x ; \nu \pm i \varepsilon)$ exists and is continuous on $R_{+}^{3} \times\left[\nu_{0}-\delta, \nu_{0}+\delta\right] \times\left[0, \varepsilon_{0}\right]$.

We now use (1.15) to obtain the asymptotic behavior of $I(x ; \nu \pm i 0)$ as $|x| \rightarrow \infty$. We set

$$
\begin{aligned}
& t_{j}=t_{j}(x, y)=j|x|-j \omega y, \quad \tilde{t}_{j}=\tilde{t}_{j}(x, y)=j|x|-j \tilde{\omega} y, \\
& Q_{j}(\omega)=\exp (-i j \pi / 2) P_{1}(j \omega)(j \omega)^{\beta}, \\
& \widetilde{Q}_{j}(\omega)=\exp (-i j \pi / 2) P_{1}(j \omega) C_{1}(j \tilde{\omega})(j \omega)^{\beta}, \\
& \tilde{\omega}=\left(\omega^{\prime},-\omega_{3}\right) .
\end{aligned}
$$


then from (1.15), (2.13)

$$
\begin{aligned}
D(x, y ; \nu \pm i \varepsilon)=(2 \pi)|x|^{-1} \sum_{j= \pm 1}[ & Q_{j}(\omega) \int_{0}^{\infty} \frac{r^{1+|\beta|} \psi(r) \exp \left(i r t_{j}\right) d r}{c r-(\nu \pm i \varepsilon)} \\
& \left.\quad-\widetilde{Q}_{j}(\omega) \int_{0}^{\infty} \frac{r^{1+|\beta|} \psi(r) \exp \left(i r \tilde{t}_{j}\right) d r}{c r-(\nu \pm i \varepsilon)}\right] \\
& +\int_{0}^{\infty} \frac{r^{2|\beta|} \psi(r) q(r x) d r}{c r-(\nu \pm i \varepsilon)}, \\
q(x)= & O\left(|x|^{-2}\right) .
\end{aligned}
$$

From Lemma 6.2 of [5] it follows that uniformly with respect to $\nu \in$ $\left[\nu_{0}-\delta, \nu_{0}+\delta\right], \varepsilon \in\left[0, \varepsilon_{0}\right]$,

$$
\int_{0}^{\infty}[c r-(\nu \pm i \varepsilon)] r^{2+|\beta|} \psi(r) q(r x) d r=O\left(|x|^{-1-\kappa}\right)
$$

$$
\kappa \in(0,1) \text { arbitrarily close to } 1 \text {. }
$$

It thus remains to evaluate

$$
\begin{aligned}
I^{ \pm}\left(t_{j}, \nu, \varepsilon\right) & \left.=\int_{0}^{\infty}[r-(\nu \pm i \varepsilon)]^{-1}\right] \phi(r) \exp \left(\text { irt }_{\jmath} / c\right) d r \\
\phi(r) & =c^{-2-|\beta|} r^{1+|\beta|} \psi(r / c)
\end{aligned}
$$

and $I^{ \pm}\left(\tilde{t}_{j}, \nu, \varepsilon\right)$. In the usual manner (cf. $\left.[5,7]\right)$ we observe that

$$
1 /[r-(\nu \pm i \varepsilon)]= \pm(2 \pi)^{1 / 2} \Phi_{1} \chi_{ \pm}(\cdot) \exp [i(\nu \pm i \varepsilon) \cdot](r)
$$

where $\chi_{ \pm}(t)$ are the characteristic functions of the half lines $R_{ \pm}$, and for a function $g_{t}(x)=g(x+t)$ we have $\Phi_{1} g_{t}(r)=\exp ($ irt $) \Phi_{1} g(r)$. By the Parseval equality for the Fourier transform we thus have from (2.17)

$$
\begin{array}{r}
I^{ \pm}\left(t_{j}, \nu, \varepsilon\right)= \pm i(2 \pi)^{1 / 2} \int_{-\infty}^{\infty} \exp \left(i r t_{j} / c\right) \Phi_{1} \chi_{ \pm}(\cdot) \exp [i(\nu \pm i \varepsilon) \cdot](r) \\
\times \Phi_{1}^{*} \Phi_{1} \phi(r) d r \\
= \pm i(2 \pi)^{1 / 2} \int_{-\infty}^{\infty} \Phi_{1}\left\{\chi_{ \pm}(\cdot) \exp [i(\nu \pm i \varepsilon)]\right\}_{t_{j} / c}(r) \Phi_{1}^{*} \Phi_{1} \phi(r) d r \\
\left.= \pm i(2 \pi)^{1 / 2} \int_{-\infty}^{\infty} \chi_{ \pm}\left(\tau+t_{j} / c\right) \exp (\nu \pm i \varepsilon)\left(\tau+t_{j} / c\right)\right] \Phi_{1} \phi(\tau) \\
= \pm i(2 \pi)^{1 / 2} \int_{-\infty}^{\infty} \chi_{ \pm}\left(t_{J} / c-G t\right) \exp \left[i(\nu \pm i \varepsilon)\left(t_{j} / c-\tau\right)\right] \Phi_{1}^{*} \phi(\tau)
\end{array}
$$


so that

(2.18) $\quad I^{+}\left(t_{j}, \nu, \varepsilon\right)$

$$
\begin{aligned}
& \quad=i(2 \pi)^{1 / 2} \int_{-\infty}^{t_{J} / c} \exp \left[i(\nu+i \varepsilon)\left(t_{j} / c-\tau\right)\right] \Phi_{1}^{*} \phi(\tau) d \tau, \\
& I^{-}\left(t_{j}, \nu, \varepsilon\right) \\
& \quad=-i(2 \pi)^{1 / 2} \int_{t_{j} / c}^{\infty} \exp \left[i(\nu-i \varepsilon)\left(t_{j} / c-\tau\right)\right] \Phi_{1}^{*} \phi(\tau) d \tau
\end{aligned}
$$

from which it follows that $I^{ \pm}\left(t_{j}(x, y), \nu, \varepsilon\right), I^{ \pm}\left(\tilde{t}_{j}(x, y), \nu, \varepsilon\right)$ are bounded and continuous for $x, y \in R_{+}^{3}, \nu \in\left[\nu_{0}-\delta, \nu_{0}+\delta\right], \varepsilon \in\left[0, \varepsilon_{0}\right]$. Now for $|x|$ large and $y$ in compact sets from $(2.15) j t_{j}(x, y), j \tilde{t}_{j}(x, y)>0$, $j= \pm 1$. Since $\phi(r)=c^{-2-|\beta|} r^{1+|\beta|} \psi(r / c)$, where $\psi(r / c)=1$ for $\left|r-\nu_{0}\right|<3 \delta$, from (2.15), (2.19) we have

(2.19) $I^{+}\left(t_{1}, \nu, 0\right)$

$$
\begin{aligned}
& \quad=i(2 \pi) c^{-2-|\beta|} \exp [i \nu(|x|-\omega y) / c] \nu^{1+|\beta|}+O\left(|x|^{-1}\right), \\
& I^{+}\left(t_{-1}, \nu, 0\right)=O\left(|x|^{-1}\right), \\
& I^{-}\left(t_{-1}, \nu, 0\right) \\
& \quad=-i(2 \pi) c^{-2-|\beta|} \exp [i \nu(-|x|+\omega y) / c] \nu^{1+|\beta|}+O\left(|x|^{-1}\right), \\
& I^{-}\left(t_{1}, \nu, 0\right)=O\left(|x|^{-1}\right),
\end{aligned}
$$

where the order relation is uniform with respect to $\nu \in\left[\nu_{0}-\delta, \nu_{0}+\delta\right]$, $\varepsilon \in\left[0, \varepsilon_{0}\right]$, and $y$ in compact sets. Similarly,

(2.20) $\quad I^{+}\left(\tilde{t}_{1}, \nu, 0\right)$

$$
\begin{aligned}
& \quad=i(2 \pi) c^{-2-|\beta|} \exp (i \nu(|x|-\tilde{\omega} y) / c] \nu^{1+|\beta|}+O\left(|x|^{-1}\right), \\
& I^{+}\left(\tilde{t}_{-1}, \nu, 0\right)=O\left(|x|^{-1}\right), \\
& I^{-}\left(\tilde{t}_{-1}, \nu, 0\right) \\
& \quad=-i(2 \pi) c^{-2-|\beta|} \exp [i \nu(-|x|+\tilde{\omega} y) / c] \nu^{1+|\beta|}+O\left(|x|^{-1}\right) \\
& I^{-}\left(\tilde{t}_{1}, \nu, 0\right)=O\left(|x|^{-1}\right) .
\end{aligned}
$$


Hence, from (2.14), (2.15), (2.17), (2.19), (2.20)

$$
\begin{aligned}
& (2.21)^{+} \quad D(x, y ; \nu+i 0) \\
& =(2 \pi)^{2} c^{-2-|\beta|}|x|^{-1} P_{1}(\omega) \nu^{1+|\beta|}\{\exp [i \nu(|x|-\omega y) / c] \\
& \left.-C_{1}(\tilde{\omega}) \exp [i \nu(|x|-\tilde{\omega} y) / c]\right\} \\
& +O\left(|x|^{-1-\kappa}\right) \\
& D(x, y ; \nu-i 0) \\
& =(2 \pi)^{2} c^{-2-|\beta|}|x|^{-1} P_{1}(-\omega) \nu^{1+|\beta|}\{\exp [i \nu(-|x|+\omega y) / c] \\
& \left.-C_{1}(-\tilde{\omega}) \exp [i \nu(-|x|+\tilde{\omega} y) / c]\right\} \\
& +O\left(|x|^{-1-\kappa}\right)
\end{aligned}
$$

and thus from (2.11), (2.13), (2.21)

$(2.21)^{-}$

$$
\begin{aligned}
& D^{\beta} v_{1}(x ; \nu \pm i 0) \\
& =(2 \pi)^{-1}|x|^{-1} P_{1}( \pm \omega) \nu^{1+|\beta|} \exp ( \pm i \nu|x| / c) \\
& \quad \times\left[\theta( \pm \omega ; \nu)-C_{1}( \pm \tilde{\omega}) \theta( \pm \tilde{\omega} ; \nu)\right]+O\left(|x|^{-1-\kappa}\right), \\
& =2 \nu^{1+|\beta|} g_{ \pm}(x ; \nu) P_{ \pm 1}(\omega)\left[\theta( \pm \omega ; \nu)-C_{\mp 1}(\omega) \theta( \pm \tilde{\omega} ; \nu)\right] \\
& \quad+O\left(|x|^{-1-\kappa}\right), \\
& \quad g_{ \pm}(x ; \nu)=(4 \pi|x|)^{-1} \exp ( \pm i \nu|x| / c), \\
& \theta(\omega ; \nu)=c^{-2-|\beta|} \omega^{\beta} \int_{R_{+}^{3}} \exp (-i \omega \nu y / c) f(y) d y, \\
& \quad \nu>0, x \in(0,1),|\beta| \geq 0 .
\end{aligned}
$$

We note that the first term on the right in (2.21) satisfies both boundary conditions $(1.4)$ and $\left(1.4^{\prime}\right)$ (see $\left.(1.7)\right)$. The $O\left(|x|^{-1-\kappa}\right)$ term thus also satisfies the boundary condition (1.4). For positive times $t \exp (-i \nu t) v_{1}(x ; \nu+i 0)$ modulo $O\left(|x|^{-1-\kappa}\right)$ is an outgoing hemispherical wave $\left(x_{3} \geq 0\right)$, while $\exp (-i \nu t) v_{1}(x ; \nu-i 0)$ is an incoming hemispherical wave.

For $\nu<0$ we see as in (2.12) that from (2.11), (2.10) for $\mu>0$

$$
D^{\beta} v_{1}(x ; \nu \pm i 0)=O\left(|x|^{-3+\mu}\right)
$$

uniformly with respect to $\nu \in\left[\nu_{0}-\delta, \nu_{0}+\delta\right], \varepsilon \in\left[0, \varepsilon_{0}\right]$.

3. $v_{-1}(\dot{x} ; \nu \pm i 0)$. Suppose first that $\nu<0$. From (1.6), (1.8), (1.10)

$$
\begin{aligned}
& \Psi_{-1}^{\prime} f(\eta)=\chi_{ \pm} \check{f}_{-1}(\eta), \\
& \check{f}_{-1}(\eta)=P_{-1}(\eta)\left[\Phi_{3} f(\eta)-C_{-1}(\tilde{\eta}) \Phi_{3} f(\tilde{\eta})\right], \\
& C_{-1}(\eta) \check{f}_{-1}(\eta)=-\check{f}_{-1}(\tilde{\eta}), \quad \tilde{\eta}=(\xi,-\rho) .
\end{aligned}
$$


Hence, from (1.11), (2.23)

$$
\begin{aligned}
& D^{\beta} v_{-1}(x ; \nu \pm i \varepsilon)=D^{\beta} \Psi_{-1}^{*}\left[\lambda_{-1}|\eta|-(\nu \pm i \varepsilon)\right]^{-1} \Psi_{-1}^{\prime} f(x) \\
& =-D^{\beta} \Psi_{-1}^{*}[c|\eta|-(|\nu| \mp i \varepsilon)]^{-1} \Psi_{-1}^{\prime} f(x) \\
& =-(2 \pi)^{-3 / 2} \int[c|\eta|-(|\nu| \mp i \varepsilon)]^{-1} \\
& \quad \times\left[\exp (i x \eta) \eta^{\beta}-\exp (i x \tilde{\eta}) \tilde{\eta}^{\beta} C_{-1}(\eta)\right] \chi_{ \pm}(\rho) \check{f}_{-1}(\eta) d \eta \\
& =-(2 \pi)^{-3 / 2} \int_{R^{3}} \exp (i x \eta) \eta^{\beta}[c|\eta|-(|\nu| \mp i \varepsilon)]^{-1} \check{f}_{-1}(\eta) d \eta .
\end{aligned}
$$

We can thus use the results of part 2 to conclude from (2.21) that for $\nu<0$.

$$
\begin{aligned}
D^{\beta} v_{-1}(x ; \nu \pm i 0) \\
=-(2 \pi)^{-1}|x|^{-1}|\nu|^{1+|\beta|} P_{-1}(\mp \omega) \exp (\mp i|\nu| x \mid) \\
\quad \times\left[\theta(\mp \omega ;|\nu|)-C_{-1}(\mp \tilde{\omega}) \theta(\mp \omega ;|\nu|)\right]+O\left(|x|^{-1-\kappa}\right) \\
=2 \nu^{1+|\beta|} g_{ \pm}(x ; \nu) P_{ \pm 1}(\omega)\left[\theta( \pm \omega ; \nu)-C_{\mp 1}(\omega) \theta( \pm \tilde{\omega} ; \nu)\right] \\
\quad+O\left(|x|^{-1-\kappa}\right),
\end{aligned}
$$

where we have used the fact that $C_{-1}(\tilde{\omega})=C_{1}(\omega)$ (see (1.7)). We again observe that the first term on the right satisfies both boundary conditions $(1.4),\left(1.4^{\prime}\right)$. The $O\left(|x|^{-1-\kappa}\right)$ term satisfies the boundary condition (1.4). For positive times (and negative frequencies) $\exp (-i \nu t) v_{-1}(x ; \nu+i 0)$ modulo $O\left(|x|^{-1-\kappa}\right)$ is an outgoing hemispherical wave, while $\exp (-i \nu t) v_{-1}(x ; \nu-i 0)$ is an incoming hemispherical wave.

For $\nu>0$, just as in (2.22),

$$
D^{\beta} v_{-1}(x ; \nu \pm i 0)=O\left(|x|^{-3+\mu}\right) .
$$

4. $v_{1}^{\prime}(x ; \nu \mp i 0)$. From (1.6), (1.8), (1.10)

$$
\begin{gathered}
\Psi_{1} f(\eta)=\chi_{\mp}(\rho) \check{f}_{1}^{\prime}(\eta), \quad \check{f}_{1}^{\prime}(\eta)=P_{1}(\eta)\left[\Phi_{3} f(\eta)-C_{1}^{\prime}(\tilde{\eta}) \Phi_{3} f(\tilde{\eta})\right], \\
C_{1}^{\prime}(\eta) \check{f}_{1}^{\prime}(\eta)=-\check{f}_{1}^{\prime}(\tilde{\eta}) .
\end{gathered}
$$

Hence, from (1.11), (2.1')

$$
\begin{aligned}
D^{\beta} v_{1}^{\prime}(x ; \nu \mp i \varepsilon)=D^{\beta}\left(\Psi_{1}^{\prime}\right)^{*}\left[\lambda_{1}(\eta)-(\nu \mp i \varepsilon)\right]^{-1} \Psi_{1} f(\eta) \\
=(2 \pi)^{-3 / 2} \int_{R^{3}}[c|\eta|-(\nu \mp i \varepsilon)]^{-1} \\
\quad \quad \times\left[\exp (i x \eta) \eta^{\beta}-\exp (i x \tilde{\eta}) \tilde{\eta}^{\beta} C_{1}^{\prime}(\eta)\right] \chi_{\mp}(\rho) \check{f}_{1}^{\prime}(\eta) d \eta \\
=(2 \pi)^{-3 / 2} \int_{R^{3}}[c|\eta|-(\nu \mp i \varepsilon)]^{-1} \exp (i x \eta) \eta^{\beta} \check{f}_{1}^{\prime}(\eta) d \eta
\end{aligned}
$$


whence as in part 2 we find for $\nu>0$

$$
\begin{aligned}
& D^{\beta} v_{1}^{\prime}(x ; \nu \mp i 0)=(2 \pi)^{-1}|x|^{-1} P_{1}(\mp \omega) \nu^{1+|\beta|} \exp (\mp i \nu|x|) \\
& \times\left[\theta(\mp \omega ; \nu)-C_{1}^{\prime}(\mp \tilde{\omega}) \theta(\mp \tilde{\omega} ; \nu)\right] \\
&+O\left(|x|^{-1-\kappa}\right) \\
&= 2 \nu^{1+|\beta|} g_{\mp}(x ; \nu) P_{\mp}(\omega)\left[S \theta(\mp \omega ; \nu)-C_{1}^{\prime}(\mp \tilde{\omega}) \theta(\mp \tilde{\omega} ; \nu)\right] \\
&+O\left(|x|^{-1-\kappa}\right) .
\end{aligned}
$$

Thus, modulo $O\left(|x|^{-1-\kappa}\right) \exp (i \nu t) v_{1}^{\prime}(x ; \nu-i 0)$ for positive $t$ is an outgoing hemispherical wave, while $\exp (i \nu t) v_{1}^{\prime}(x ; \nu+i 0)$ is an incoming wave. The first term on the right satisfies both boundary conditions (1.4), $\left(1.4^{\prime}\right)$, and the $O\left(|x|^{-1-\kappa}\right)$ term thus satisifies the boundary condition $\left(1.4^{\prime}\right)$.

Just as in (2.22), for $\nu<0$

$$
D^{\beta} v_{1}^{\prime}(x ; \nu \mp i 0)=O\left(|x|^{-3+\mu}\right) .
$$

5. $v_{-1}^{\prime}(x ; \nu \mp i 0)$. From (1.6), (1.8), (1.10)

$$
\begin{aligned}
& \Psi_{-1} f(\eta)=\chi_{ \pm}(\rho) \check{f}_{-1}^{\prime}(\eta), \\
& \check{f}_{-1}^{\prime}(\eta)=P_{-1}(\eta)\left[\Phi_{3} f(\eta)-C_{-1}^{\prime}(\tilde{\eta}) \Phi_{3} f(\tilde{\eta})\right], \\
& C_{-1}^{\prime}(\eta)=-\check{f}_{-1}^{\prime}(\tilde{\eta}) .
\end{aligned}
$$

Hence, from (1.11), $\left(2.1^{\prime}\right)$ for $\nu<0$.

$$
\begin{aligned}
D^{\beta} v_{-1}^{\prime}(x ; \nu \mp i 0) & =D^{\beta}\left(\Psi_{-1}^{\prime}\right)^{*}\left[\lambda_{-1}(\eta)-(\nu \mp i 0)\right]^{-1} \Psi_{-1} f(\eta) \\
& =-D^{\beta}\left(\Psi_{-1}^{\prime}\right)^{*}[c|\eta|-(|\nu| \pm i 0)]^{-1} \Psi_{-1} f(\eta),
\end{aligned}
$$

so that as in part 4

$$
\begin{aligned}
D^{\beta} v_{-1}^{\prime}(x ; \nu \mp i 0)= & 2 \nu^{1+|\beta|}(-1)^{|\beta|} P_{1}(\mp \omega) g_{\mp}(x ; \nu) \\
& \times\left[\theta(\mp \omega ; \nu)-C_{1}^{\prime}( \pm \omega) \theta(\mp \tilde{\omega} ; \nu)\right] \\
& +O\left(|x|^{-1-\kappa}\right) .
\end{aligned}
$$

For $\nu>0$, just as in (2.22),

$$
D^{\beta} v_{-1}^{\prime}(x ; \nu \mp i 0)=O\left(|x|^{-3+\mu}\right) .
$$

6a. $v_{S}(x ; \nu \pm i 0), v_{S}^{\prime}(x ; \nu \pm i 0), S=E, M$. The case $\alpha_{1}>0$. These components are all estimated in the same way. We consider, for example, $v_{E}(x ; \nu \pm i 0)$. From $(1.9)-(1.11)$, since $e(\xi)$ is in the 
lower half plane and $0 \neq \nu \in R$, we can immediately write

$$
\begin{aligned}
v_{E}(x ; \nu & \pm i 0)=\Sigma_{E}^{*}[e(\cdot)-(\nu \pm i 0)]^{-1} \Sigma_{E}^{\prime} f(x) \\
= & i \mu q(c \alpha \varepsilon)^{-1}(2 \pi)^{-1} \int_{R^{2}} \exp \left[i x^{\prime} \xi+\tau_{e}(\xi) x_{3}\right]|\xi|^{-3} \\
& \times \mathscr{E}(\xi)^{t} \mathscr{E}(\xi)[e(\xi)-\nu]^{-1} d \xi E \int_{0}^{\infty} \exp \left[i \tau_{e}(\xi) y_{3}\right] \Phi_{3} f\left(\xi, y_{3}\right) d y_{3} .
\end{aligned}
$$

Setting $h|\xi|=\operatorname{Im} \tau_{e}(\xi)\left(\right.$ see (1.9)) and noting that $\inf _{\xi}|e(\xi)-\nu| \geq \delta>$ 0 , we have just as in (2.7)

$$
\begin{aligned}
& \left|x_{3}^{2} v_{E}(x ; \nu \pm i 0)\right| \\
& \quad \leq c(\nu, f) x_{3}^{2} \partial_{3}^{2} \int_{a}^{b} \int_{0}^{\infty} \exp \left[-h|\xi|\left(x_{3}+y_{3}\right)\right] d|\xi| d y_{3} \\
& \quad \leq c(f)<\infty
\end{aligned}
$$

We can thus estimate $v(x ; \nu \pm i 0)$ in exactly the same way as $R(x)$ in part 1. Estimating $D^{\beta} v(x ; \nu \pm i 0)$ in a similar way, we thus have

(2.30) $|x|^{2}\left|D^{\beta} v_{S}(x ; \nu \pm i 0)\right| \leq c(\nu, f)<\infty$,

$$
|x|^{2}\left|D^{\beta} v_{S}^{\prime}(x ; \nu \pm i 0)\right| \leq c(\nu, f)<\infty, \quad S=E, M,|\beta| \geq 0,
$$

$$
|x| \rightarrow \infty, x_{3} \geq 0 \text {. }
$$

$6 \mathrm{~b}^{+}$. The case $\alpha-i \alpha_{2}, \alpha_{2}>0$. In this case $p=\sqrt{\left(\mu / \varepsilon+\alpha_{2}^{2}\right)^{-1}}$ $q=\alpha_{2} p>0$, and $e(\xi)=c q|\xi|$ lies on the positive semiaxis, while $m(\xi)=-p|\xi| / \varepsilon$ lies on the negative semiaxis (this follows from (1.5), (1.9), and the condition that $\operatorname{Im} \tau_{e, m} \geq 0$ ). This situation is responsible for the fact that the surface waves decay only like $|x|^{-1 / 2}$ along the boundary $\left\{x_{3}=0\right\}$. We first consider the case $\zeta=\nu \pm i \varepsilon, \nu>0$, $\varepsilon \in\left(0, \varepsilon_{0}\right]$; we choose $\delta>0$ so that $\nu_{0}-5 \delta>0, \nu \in\left(\nu_{0}-\delta, \nu_{0}+\delta\right)$. Let $\psi \in C_{0}^{\infty}(R)$, supp $\psi=\left\{\left|e(r)-\nu_{0}\right| \leq 4 \delta\right\}=\left\{\left|c q r-\nu_{0}\right|<4 \delta\right\} \subset$ $(0, \infty), \psi(r)=1$ for $\left|c q r-\nu_{0}\right|<3 \delta$, and let $\chi \in C_{0}^{\infty}\left(R^{2} \backslash\{0\}\right)$, $\chi(\xi) \equiv \chi(r s) \equiv \psi(r), s \in S^{1}$.

$$
\begin{aligned}
D^{\beta} v_{E}(x ; \zeta)= & D^{\beta} \Sigma_{E}^{*}[e(\cdot)-\zeta]^{-1} \Sigma_{E} f(x) \\
= & D^{\beta} \Sigma_{E}^{*}[e(\cdot)-\zeta]^{-1} \chi(\cdot) \Sigma_{E} f(x) \\
& +D^{\beta} \Sigma_{E}^{*}[e(\cdot)-\zeta]^{-1}[1-\chi(\cdot)] \Sigma_{E} f(x) \\
\equiv & I_{E}(x ; \zeta)+J_{E}(x ; \zeta) .
\end{aligned}
$$

Since for $\xi \in \operatorname{supp}(1-\chi)$ we have $|e(\xi)-\zeta| \geq 3 \delta$, just as in $6 \mathrm{a}$, $J_{E}(x ; \nu \pm i 0)$ exists, is continuous on $R_{+}^{3} \times\left[\nu_{0}-\delta_{0}, \nu+\delta\right] \times\left[0, \varepsilon_{0}\right]$, and

$$
|x|^{2}\left|J_{E}(x ; \nu \pm i 0)\right| \leq c(\nu, f)<\infty, \quad x_{3} \geq 0,|x| \rightarrow \infty .
$$


With $h=\mu c q / \alpha_{2}, \beta=\beta^{\prime} \beta_{3}$, and $c_{e}=(2 \pi)^{-2} \mu q(i h)^{\beta} 3\left(\alpha_{2} c \varepsilon\right)^{-1}$ (2.33) $I_{E}(x ; \zeta)$

$$
\begin{aligned}
& =c_{e} \int_{R^{2}} d \xi \int_{R_{+}^{3}} d y \xi^{\beta^{\prime}} \exp \left(i x^{\prime} \xi-h|\xi| x_{3}\right)|\xi|^{-3+\beta_{3}} \mathscr{E}(\xi)^{\bar{t}} \mathscr{E}(\xi) \\
& \quad \times[e(\xi)-\zeta]^{-1} \chi(\xi) \exp \left(-i y^{\prime} \xi-h y_{3}\right) E f(y) \\
& \equiv c_{e} \int_{R^{3}} D(x, y ; \zeta) E f(y) d y,
\end{aligned}
$$

where with $\xi=r s \equiv r\left(\cos \phi_{\xi}, \sin \phi_{\xi}\right)$

$$
\begin{aligned}
D(x, y ; \zeta)=\int_{0}^{\infty} d r(c q r & -\zeta)^{-1} \psi(r) r^{2+|\beta|} \exp \left[-h r\left(x_{3}+y_{3}\right)\right] \\
& \times \int_{S^{1}}\left\{s^{\beta^{\prime}} \mathscr{E}(s)^{\bar{t}} \mathscr{E}(s) \exp \left[\operatorname{irs}\left(x^{\prime}-y^{\prime}\right)\right]\right\} d \phi .
\end{aligned}
$$

Since $\psi(r)=1$ for $\left|c q r-\nu_{0}\right| \leq 2 \delta$, replacing this interval by a semicircle in the lower $(\zeta=\nu+i \varepsilon)$ or upper $(\zeta=\nu-i \varepsilon)$ half plane and extending $\psi$ to the half disk by one, it follows that $I_{E}(x ; \nu \pm i 0)$ exists and is continuous on $R_{+}^{3} \times\left[\nu_{0}-\delta, \nu_{0}+\delta\right]$. Hence, $D^{\beta} v_{E}(x ; \nu \pm i \varepsilon)$ exists and is continuous on $R_{+}^{3} \times\left[\nu_{0}-\delta, \nu_{0}+\delta\right] \times\left[0, \varepsilon_{0}\right]$.

We now estimate $D(x, y ; \nu \pm i \varepsilon)$ for large $\left|x^{\prime}\right|$ and fixed $x_{3} \in R_{+}^{3}$. With $x^{\prime}=\left|x^{\prime}\right| \gamma, \gamma=\left(\cos \phi_{x}, \sin \phi_{x}\right)$ from (1.16)

$$
\begin{aligned}
& \int_{S^{1}}\left\{\exp \left(i r\left|x^{\prime}\right| \gamma s\right) \exp \left(-i r s y^{\prime}\right) s^{\beta^{\prime}} \mathscr{E}(s)^{\bar{t} \mathscr{E}(s) d \phi\}}\right. \\
& =(2 \pi)^{1 / 2}\left|r x^{\prime}\right|^{-1 / 2} \sum_{j= \pm 1} \exp \left[i j r\left(\left|x^{\prime}\right|-\gamma y^{\prime}\right)-i j \pi / 4\right]
\end{aligned}
$$

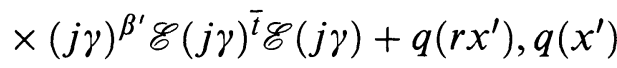

$$
\begin{aligned}
& =O\left(\left|x^{\prime}\right|^{-3 / 2}\right) \text {. }
\end{aligned}
$$

and hence

$$
\begin{aligned}
D(x, y ; \zeta)= & \int_{0}^{\infty} d r(c q r-\zeta)^{-1} \psi(r) r^{2+|\beta|} \\
& \times \exp \left[-h r\left(x_{3}+y_{3}\right)\right] \\
& \times\left\{(2 \pi)^{1 / 2}\left|r x^{\prime}\right|^{-1 / 2}\right. \\
& \times \sum_{j= \pm 1} \exp \left[i j r\left(\left|x^{\prime}\right|-\gamma y^{\prime}\right)-i j \pi / 4\right] \\
& \left.\times(j \gamma)^{\beta^{\prime}} \mathscr{E}(j \gamma)^{\bar{t}}(j \gamma)+q\left(r x^{\prime}\right)\right\} \\
\equiv & D_{1}(x, y ; \zeta)+D_{2}(x, y ; \zeta),
\end{aligned}
$$


where

$$
\begin{aligned}
& D_{2}(x, y ; \zeta) \\
& \quad=\int_{0}^{\infty} d r(c q r-\zeta)^{-1} \psi(r) r^{2+|\beta|} \exp \left[-h r\left(x_{3}+y_{3}\right)\right] q\left(r x^{\prime}\right) .
\end{aligned}
$$

Repeating essentially the proof of Lemma 6.2 of [5], we have

$$
\begin{gathered}
\left|D_{2}(x, y ; \nu \pm i \varepsilon)\right| \leq c\left(\nu_{0}, \delta, f\right) \exp \left(-a^{\prime} x_{3}\right)\left(1+x_{3}\right)\left|x^{\prime}\right|^{-1-\kappa^{\prime}} \\
\left|x^{\prime}\right| \geq R, x_{3} \geq 0, \kappa^{\prime} \in(0,1 / 2) \text { arbitrarily close to } 1 / 2 \\
a^{\prime}=\mu\left(\nu_{0}-4 \delta\right) / \alpha_{2}>0
\end{gathered}
$$

uniformly with respect to $\nu \in\left[\nu_{0}-\delta, \nu_{0}+\delta\right], \varepsilon \in\left[0, \varepsilon_{0}\right]$.

From (2.35)

$$
\begin{aligned}
& D_{1}(x, y ; \zeta) \\
& =(2 \pi)^{1 / 2}\left|x^{\prime}\right|^{-1 / 2} \sum_{j= \pm 1} \exp (-i j \pi / 4)(j \gamma)^{\beta} \mathscr{E}(j \gamma)^{\bar{t}} \mathscr{E}(j \gamma) \\
& \times \int_{0}^{\infty} d r(c q r-\zeta)^{-1} \psi(r) r^{3 / 2+|\beta|} \exp \left[-h r\left(x_{3}+y_{3}\right)\right] \\
& \times \exp \left[i j r\left(\left|x^{\prime}\right|-\gamma y^{\prime}\right)\right] \\
& \equiv(2 \pi)^{1 / 2}\left|x^{\prime}\right|^{-1 / 2} \sum_{j= \pm 1} \exp (-i j \pi / 4)(j \gamma)^{\beta^{\prime}} \mathscr{E}(j \gamma)^{\bar{t}} \mathscr{E}(j \gamma) \\
& \times I^{ \pm}\left(t_{j}, x_{3}, y_{3} ; \nu, \varepsilon\right) \\
& I^{ \pm}\left(t_{j}, x_{3}, y_{3} ; \zeta\right)=\int_{0}^{\infty} d r(r-\zeta)^{-1} \phi\left(r, x_{3}, y_{3}\right) \exp \left(\operatorname{irt}_{j}\right) \\
& \zeta=\nu \pm i \varepsilon, \quad t_{j}=j\left(\left|x^{\prime}\right|-\gamma y^{\prime}\right) / c q, \quad j= \pm 1, \\
& \phi\left(r, x_{3}, y_{3}\right)=(c q)^{-5 / 2-|\beta|} \psi(r / c q) \exp \left[-\mu r\left(x_{3}+y_{3}\right) / \alpha_{2}\right] r^{3 / 2+|\beta|} \\
& \equiv \lambda(r) \exp \left[-\mu r\left(x_{3}+y_{3}\right) / \alpha_{2}\right] \text {, } \\
& \operatorname{supp} \lambda(r) \subset\left[\nu_{0}-4 \delta, \nu_{0}+4 \delta\right] \text {. }
\end{aligned}
$$

Hence,

(2.38) $\quad I^{+}\left(t_{j}, x_{3}, y_{3} ; \nu, \varepsilon\right)$

$$
\begin{aligned}
& \quad=i(2 \pi)^{1 / 2} \int_{-\infty}^{t_{j}} \exp \left[i(\nu+i \varepsilon)\left(t_{j}-\tau\right)\right] \Phi_{1}^{*} \phi\left(\tau, x_{3}, y_{3}\right) d \tau, \\
& I^{-}\left(t_{j}, x_{3}, y_{3} ; \nu, \varepsilon\right) \\
& \quad=-i(2 \pi)^{1 / 2} \int_{t_{j}}^{\infty} \exp \left[i(\nu-i \varepsilon)\left(t_{j}-\tau\right)\right] \Phi_{1}^{*} \phi\left(\tau, x_{3}, y_{3}\right) d \tau,
\end{aligned}
$$


from which it follows that $I^{ \pm}\left(t_{j}, x_{3}, y_{3} ; \nu, \varepsilon\right)$ are bounded and continuous for $x_{3}, y_{3} \in R_{+}^{3}, \nu \in\left[\nu_{0}-\delta_{0}, \nu_{0}+\delta\right], \varepsilon \in\left[0, \varepsilon_{0}\right]$. With $\tilde{a}=\nu_{0}-5 \delta>0, r-\tilde{a}>0$ for $r \in \operatorname{supp} \lambda$

$$
\begin{aligned}
& \Phi_{1}^{*} \phi\left(\tau, x_{3}, y_{3}\right)=(2 \pi)^{-1 / 2} \int e^{i r \tau} \lambda(r) \exp \left[-h r\left(x_{3}+y_{3}\right) / c q\right] d r \\
& \equiv \exp \left[-\mu \tilde{a}\left(x_{3}+y_{3}\right) / \alpha_{2}\right] \pi\left(\tau, x_{3}, y_{3}\right), \\
& \pi\left(\tau, x_{3}, y\right)=(2 \pi)^{-1 / 2} \int e^{i r \tau} \lambda(r) \exp \left[-\mu(r-\tilde{a})\left(x_{3}+y_{3}\right) / \alpha_{2}\right] d r .
\end{aligned}
$$

It follows readily on integrating by parts that $\tau^{2}\left|\pi\left(\tau, x_{3}, y_{3}\right)\right| \leq c\left(\nu_{0}\right)$. Hence, from (2.38), (2.39)

$$
\begin{aligned}
& (2.40) I^{ \pm}\left(t_{ \pm 1}, x_{3}, y_{3} ; \nu, \varepsilon\right) \\
& = \pm(2 \pi) \exp \left(i \nu t_{ \pm 1}\right) \phi(\nu)+\exp \left(-\mu \tilde{a} x_{3} / \alpha_{2}\right) q^{ \pm}\left(x^{\prime}, y\right), \\
& \left|q^{ \pm}\left(x^{\prime}, y\right)\right| \leq c^{ \pm}\left(\nu_{0}\right)\left|x^{\prime}\right|^{-1}, \quad\left|x^{\prime}\right| \rightarrow \infty \text {. } \\
& I^{ \pm}\left(t_{\mp 1}, x_{3}, y_{3} ; \nu, 0\right)=\exp \left(-\mu \tilde{a} x_{3} / \alpha_{2}\right) \tilde{q}^{ \pm}\left(x^{\prime}, y\right), \\
& \left|\tilde{q}^{ \pm}\left(x^{\prime}, y\right)\right| \leq \tilde{c}^{ \pm}\left(\nu_{0}\right)\left|x^{\prime}\right|^{-1}, \quad\left|x^{\prime}\right| \rightarrow \infty, \tilde{a}=\nu_{0}-5 \delta>0 \text {. }
\end{aligned}
$$

Hence, from (2.37)

$$
\begin{aligned}
& D_{1}(x, y ; \nu \pm i 0) \\
& = \pm i(2 \pi)^{3 / 2}\left|x^{\prime}\right|^{-1 / 2} \exp (\mp i \pi / 4) \exp \left(i \nu t_{\mp}\right) \lambda(n)(j \gamma)^{\beta^{\prime}} \\
& \quad \times \mathscr{E}( \pm \gamma)^{\bar{t}}( \pm \gamma)+Q^{ \pm}(x, y), \\
& \left|Q^{ \pm}(x, y)\right| \leq c^{ \pm}\left(\nu_{0}, \beta\right) \exp \left(-a x_{3}\right)\left|x^{\prime}\right|^{-3 / 2}, \quad \begin{array}{r}
a= \\
\mu\left(\nu_{0}-5 \delta\right) / \alpha_{2}, \\
x_{3}>0,\left|x^{\prime}\right| \rightarrow \infty,
\end{array}
\end{aligned}
$$

and from this and $(2.35),(2.36)$ we have

(2.41) $D(x, y ; \nu \pm i 0)$

$$
\begin{aligned}
& =c^{ \pm}(\nu)\left|x^{\prime}\right|^{-1 / 2} \exp \left[ \pm i \nu\left(\left|x^{\prime}\right|-\gamma y^{\prime}\right) / c q-\mu \nu\left(x_{3}+y_{3}\right) / \alpha_{2}\right] \\
& \times( \pm \gamma)^{\beta^{\prime}} \mathscr{E}( \pm \gamma)^{\bar{t}} \mathscr{E}( \pm \gamma)+P^{ \pm}(x, y, \nu, \beta) . \\
& c^{ \pm}(\nu)= \pm i(2 \pi)^{3 / 2}(c q)^{-1}(\nu / c q)^{3 / 2+|\beta|} \exp (\mp(i \pi / 4)) \text {, } \\
& \left|P^{ \pm}(x, y ; \nu, \beta)\right| \leq c\left(\nu_{0}, \beta, f\right) \exp \left(-a x_{3}\right)\left|x^{\prime}\right|^{-1-\kappa^{\prime}}, \\
& \left|x^{\prime}\right| \text { large }, \quad x_{3} \geq 0, \quad a=\mu\left(\nu_{0}-5 \delta\right)>0 \text {, } \\
& \kappa^{\prime} \in(0,1 / 2) \text { arbitrarily close to } 1 / 2 \text {. }
\end{aligned}
$$


Thus, from (2.31), (2.32), (2.33), (2.41)

$$
\begin{aligned}
& D^{\beta} v_{E}(x ; \nu \pm i 0) \\
& =c_{E}^{ \pm}(\nu, \beta) \exp \left( \pm i \nu\left|x^{\prime}\right| / c q-\mu \nu x_{3} / \alpha_{2}\right)\left|x^{\prime}\right|^{-1 / 2 \mathscr{E}( \pm \gamma)} \\
& \times^{\bar{t} \mathscr{E}}( \pm \gamma) \theta_{E}( \pm \gamma, \nu)+R_{E}^{ \pm}\left(x, \nu, \beta, \quad \nu>0, \alpha_{2}>0,\right. \\
& \theta_{E}( \pm \gamma, \nu)=(\gamma)^{\beta^{\prime}} \int_{R^{3}} \exp \left(\mp i \nu \gamma y^{\prime} / c q-\mu \nu y_{3} / \alpha_{2}\right) E f(y) d y \text {, } \\
& \left|R_{E}^{ \pm}(x, \nu, \beta)\right| \leq c\left(\nu_{0}, \beta ; f\right) \exp \left(-a x_{3}\right)\left(1+x_{3}\right)\left|x^{\prime}\right|^{-1-\kappa^{\prime}} . \\
& c_{E}^{ \pm}(\nu, \beta)= \pm i(2 \pi)^{-1 / 2} \mu\left(\alpha_{2} c^{2} \varepsilon\right)^{-1}(\nu / c q)^{3 / 2+\beta}\left(i \mu c q / \alpha_{2}\right)^{\beta_{3}} \\
& \times \exp (\mp i \pi / 4) \text {, } \\
& a=\mu\left(v_{0}-5 \delta\right) / \alpha_{2}>0, \quad \kappa^{\prime} \in(0,1 / 2) \text { arbitrarily close to } 1 / 2, \\
& x^{\prime}=\left|x^{\prime}\right| \gamma, x_{3} \geq 0, \quad\left|x^{\prime}\right| \text { large, }
\end{aligned}
$$

uniformly with respect to $\nu \in\left[\nu_{0}-\delta, \nu_{0}+\delta\right], \nu_{0}-5 \delta>0, \delta=\delta\left(\nu_{0}\right)$.

REMARK 1. Writing $v_{E}(x ; \nu \pm i 0)=\stackrel{\circ}{v}_{E}(x ; \nu \pm i 0)+R_{E}^{ \pm}(x ; \nu)$, we see that $\exp (-i \nu t) \stackrel{\circ}{E}_{E}(x ; \nu-i 0)$ for $t>0$ and each $x_{3} \geq 0$ is an outgoing cylindrical wave, while $\exp (-i \nu t) \stackrel{\circ}{v}_{E}(x ; \nu-i 0)$ is an incoming cylindrical wave. Each decays like $\left|x^{\prime}\right|^{-1 / 2}$ for $x_{3}=0$. The leading term $\stackrel{\circ}{v}_{E}(x ; \nu \pm i 0)$ satisfies the boundary condition $(1.4)$ and hence so also does $R_{E}^{ \pm}(x ; \nu)$. Further, $[\Lambda(D)-\nu I] \stackrel{\circ}{v}(x ; \nu \pm i 0)=0$ modulo a term decaying like $\exp \left(-\mu \nu x_{3} / \alpha_{2}\right)\left|x^{\prime}\right|^{-3 / 2}$, and hence $[\Lambda(D)-\nu I] v_{E}(x ; \nu \pm$ $i 0) \in \mathscr{X}$ as it must, since $[\Lambda(D)-\nu I] v_{E}(x ; \nu \pm i 0)=\Sigma_{E}^{*} \Sigma_{E} f \in \mathscr{X}$ (see (2.31)). We observe also that with $A(D)$ of $(0.1)$ for $\gamma \in S^{1}$

$$
\bar{t}_{\mathscr{E}}( \pm \gamma) A(\gamma, 0) \mathscr{E}( \pm \gamma)= \pm 2 c q / \mu .
$$

From (1.9)-(1.11) we have

$$
\begin{aligned}
D^{\beta} v_{M}(x ; \zeta)=D^{\beta} \Sigma_{M}^{*}[m(\cdot)-\zeta]^{-1} \Sigma_{M} f(x) & \\
= & c_{m} \int_{R^{3}} \int_{R^{2}}\left\{\exp \left(i x^{\prime} \xi-\alpha_{2} p|\xi| x_{3}\right)(\xi)^{\beta^{\prime}}|\xi|^{-3+\beta_{3}} \mathscr{M}(\xi)\right. \\
& \times^{\bar{t}} \mathscr{M}(\xi)[m(\xi)-\zeta]^{-1} \exp \left(-i \xi y^{\prime}-\alpha_{2} p|\xi| x_{3}\right) E f(y) d \xi d y, \\
m(\xi)= & -p|\xi| / \varepsilon, \quad c_{m}=(2 \pi)^{-2} p \alpha_{2} \varepsilon\left(i p \alpha_{2}\right)^{\beta_{3}}, \quad \zeta=\nu \pm i \varepsilon .
\end{aligned}
$$

Since $p>0$ for $\alpha_{2}>0$, in the case $\nu>0$ we have $|m(\xi)-\zeta| \geq$ const $>$ 0 , and, as in $6 \mathrm{a}, D^{\beta} v_{M}(x ; \nu \pm i 0)$ exists and is continuous on $R_{+}^{3} \times$ $\left[\nu_{0}-\delta, \nu_{0}+\delta\right] \times\left[0, \varepsilon_{0}\right]$, and for $|x|$ large

$$
|x|^{2}\left|D^{\beta} v(x ; \nu \pm i 0)\right| \leq c(\nu, f)<\infty, \quad \nu>0, \quad \alpha_{2}>0 .
$$


In the case $\nu<0,|e(\xi)-\zeta| \geq$ const $>0$, so the same argument applies to $D^{\beta} v_{E}(x ; \zeta)$, and for large $|x|$

$$
|x|^{2} D^{\beta} v_{E}(x ; \nu \pm i 0) \mid \leq c(\nu, f)<\infty, \quad \nu<0, \alpha_{2}>0 .
$$

As for $D^{\beta} v_{M}(x ; \zeta), \zeta=\nu \pm i \varepsilon, \nu<0$, we proceed from (2.43) exactly as above in (2.31)-2.42) and conclude that $D^{\beta} v_{M}(x ; \nu \pm i \varepsilon)$ exists and is continuous on $R_{+}^{3} \times\left[\nu_{0}-\delta, \nu_{0}+\delta\right] \times\left[0, \varepsilon_{0}\right]$, and for $x^{\prime}=\left|x^{\prime}\right| \gamma,\left|x^{\prime}\right|$ large, $\nu \in\left[\nu_{0}-\delta, \nu_{0}+\delta\right] \subset(-\infty, 0),\left|\nu_{0}\right|-5 \delta>0$

$$
\begin{aligned}
& \quad D^{\beta} v_{M}(x ; \nu \pm i 0) \\
& =c_{M}^{ \pm}(\nu, \beta)\left|x^{\prime}\right|^{-1 / 2} \exp \left( \pm i \nu \varepsilon\left|x^{\prime}\right| / p+\alpha_{2} \varepsilon \nu x_{3}\right) \mathscr{M}(\mp \gamma) \\
& \quad{ }^{\bar{t}} \mathscr{M}(\mp \gamma) \theta_{M}(\mp \gamma ; \nu)+R_{M}^{ \pm}(x ; \nu), \quad \nu<0, \alpha_{2}>0, \\
& \theta_{M}(\mp \gamma ; \nu)=\int_{R_{+}^{3}} \exp \left( \pm i \nu \varepsilon \gamma y^{\prime} / p+\alpha_{2} \varepsilon \nu y_{3}\right) E f(y) d y . \\
& \left|R_{+}^{ \pm}(x ; \nu)\right| \\
& \leq c(\nu, f, \beta) \exp \left(-b x_{3}\right)\left(1+x_{3}\right)\left|x^{\prime}\right|^{-1-\kappa^{\prime}}, \quad \kappa^{\prime} \in(0,1 / 2), \\
& \quad b=\alpha_{2} \varepsilon_{0}(|\nu|-5 \delta)>0, \\
& c_{M}^{ \pm}= \pm i(2 \pi)^{-1 / 2} \alpha_{2} \varepsilon^{2}(\varepsilon|\nu| / p)^{3 / 2+|\beta|}\left(i \alpha_{2} p\right)^{\beta_{3}} \exp ( \pm i \pi / 4) .
\end{aligned}
$$

REMARK 2. The full analogue of Remark 1 holds for $v_{M}(x ; \nu \pm i 0)$, $\nu<0$. In particular,

$$
{ }^{\bar{t}} \mathscr{M}(\mp \gamma) A(\gamma, 0) \mathscr{M}(\mp \gamma)= \pm 2 p / \varepsilon^{2} .
$$

Further, from (1.14) with $t=0$ and $S=\{0, \pm 1, E, M\}$

$$
\begin{aligned}
f & =\Pi_{0} f+\Psi_{1}^{*} \Psi_{1} f+\Psi_{-1}^{*} \Psi_{-1} f+\Sigma_{E}^{*} \Sigma_{E}^{*} f+\Sigma_{M}^{*} \Sigma_{M} f \\
& =\sum_{j \in S} \Pi_{j} f
\end{aligned}
$$

where the $\Pi_{j}$ are mutually orthogonal orthoprojectors in $\mathscr{X}[2,3]$. Thus, writing $v_{R}(x ; \nu \pm i 0)=v_{0}(x ; \nu)+v_{1}(x ; \nu \pm i 0)+v_{-1}(x ; \nu+i 0)$ for the "Raumwelle" and $v_{S}(x ; \nu \pm i 0)=v_{E}(x ; \nu \pm i 0)+v_{M}(x ; \nu \pm i 0)$ for the surface wave, we see in all cases from (2.2) that

$$
\mathscr{X} \ni[\Lambda(D)-\nu I] v_{R}(x ; \nu \pm i 0)=\sum_{j=0, \pm 1} \Pi_{j} f(x)
$$

is orthogonal to

$$
\mathscr{X} \ni[\Lambda(D)-\nu I] v_{S}(x ; \nu \pm i 0)=\Pi_{E} f(x)+\Pi_{M} f(x) .
$$


$6 \mathrm{~b}^{-}$. The case $\alpha=i \alpha_{2}, \alpha_{2}<0$. In this case $p=-\sqrt{\left(\mu / \varepsilon+\alpha_{2}\right)}<0$ and $q=-\alpha_{2} p<0$, so $m(\xi)=-p|\xi| / \varepsilon \in[0, \infty), e(\xi)=c q|\xi| \in(-\infty, 0]$ (this follows from (1.5), (1.9) and the condition $\operatorname{Im} \tau_{e, m} \geq 0$ ); the roles of the TE- and TM-waves are thus reversed. Mutatis mutandis, however, the arguments are exactly the same as in $6 b^{+}$, so we shall simply state the results.

For $\nu>0 D^{\beta} v_{M, E}(x ; \nu \pm i \varepsilon)$ exist and are continuous on $R_{+}^{3} \times$ $\left[\nu_{0}-\delta, \nu_{0}+\delta\right] \times\left[0, \varepsilon_{0}\right], \nu_{0}-5 \delta>0$. For large $\left|x^{\prime}\right|$ and $x_{3} \geq 0$

$$
\begin{aligned}
D^{\beta} & v_{M}(x ; \nu \pm i 0) \\
= & \tilde{c}_{M}^{ \pm}(\nu, \beta)\left|x^{\prime}\right|^{-1 / 2} \exp \left( \pm i \nu \varepsilon\left|x^{\prime}\right| /|p|-\left|\alpha_{2}\right| \varepsilon \nu x_{3}\right) \mathscr{M}( \pm \gamma) \\
& \times{ }^{\bar{t}} \mathscr{M}( \pm \gamma) \tilde{\theta}_{M}( \pm \gamma ; \nu)+\widetilde{R}_{M}^{ \pm}(x ; \nu), \quad \nu>0, \alpha_{2}<0
\end{aligned}
$$$$
\tilde{\theta}_{M}( \pm \gamma ; \nu)=\int_{R^{3}} \exp \left(\mp i \nu \varepsilon \gamma y^{\prime} /|p|-\left|\alpha_{2}\right| \varepsilon \nu y_{3}\right) E f(y) d y
$$

$$
\left|R_{M}^{ \pm}(x ; \nu)\right| \leq c\left(\nu_{0}, f\right) \exp \left(-b x_{3}\right)\left|x^{\prime}\right|^{-1-\kappa^{\prime}}
$$

$$
\begin{aligned}
\tilde{c}_{M}(\nu, \beta) & = \pm i(2 \pi)^{1 / 2}\left|\alpha_{2}\right|\left(i \alpha_{2} p\right)^{\beta} 3(\varepsilon \nu /|p|)^{3 / 2+|\beta|} \exp (\mp i \pi / 4) \\
b & =\left|\alpha_{2}\right| \varepsilon\left(\nu_{0}-5 \delta\right)>0, \quad \kappa^{\prime} \in(0,1 / 2)
\end{aligned}
$$

$$
|x|^{2}\left|D^{\beta} v_{E}(x ; \nu \pm i 0)\right| \leq c\left(\nu_{0}, f\right)<\infty, \quad \nu>0, \alpha_{2}<0
$$

For $\nu<0 D^{\beta} v_{M, E}(x ; \nu \pm i \varepsilon)$ exist and are continuous on $R_{+}^{3} \times$ $\left[\nu_{0}-\delta, \nu_{0}+\delta\right] \times\left[0, \varepsilon_{0}\right],\left|\nu_{0}\right|-5 \delta>0$. For large $\left|x^{\prime}\right|$ and each $x_{3} \geq 0$

(2.51) $D^{\beta} v_{E}(x ; \nu \pm i 0)$

$$
\begin{aligned}
& =\tilde{c}_{E}^{ \pm}(\nu, \beta) \exp \left( \pm i \nu\left|x^{\prime}\right| / c|q|+\mu \nu x_{3} /\left|\alpha_{2}\right|\right)\left|x^{\prime}\right|^{-1 / 2} \\
& \times \mathscr{E}(\mp \gamma)^{\bar{t}} \mathscr{E}(\mp \gamma) \tilde{\theta}_{E}(\mp \gamma, \beta)+\widetilde{R}_{E}^{ \pm}(x, \nu, \beta),
\end{aligned}
$$

$$
\nu<0, \alpha_{2}<0 \text {, }
$$

$$
\begin{gathered}
\tilde{\theta}_{E}(\mp \gamma, \nu)=(\mp \gamma)^{\beta^{\prime}} \int \exp \left(\mp i \nu \gamma y^{\prime} / c|q|+\mu \nu y_{3} /\left|\alpha_{2}\right|\right) E f(y) d y \\
\left.\left|\widetilde{R}_{E}^{ \pm}(x ; \nu, \beta) \leq c\left(\nu_{0}, \beta, f\right) \exp \left(-a x_{3}\right)\left(1+x_{3}\right)\right| x^{\prime}\right|^{-1-\kappa^{\prime}} \\
a=\mu\left(\left|\nu_{0}\right|-5 \delta\right) /\left|\alpha_{2}\right|>0, \quad \kappa^{\prime} \in(0,1 / 2) .
\end{gathered}
$$




$$
|x|^{2}\left|D^{\beta} v_{M}(x ; \nu \pm i 0)\right| \leq c\left(\nu_{0}, \beta, f\right)<\infty, \quad \nu<0, \alpha_{2}<0 .
$$

The results of this section can now be summarized as follows.

THEOREM 2.1. For any $0 \neq \nu \in R$ and $f \in C_{0}^{\infty}\left(R_{+}^{3}\right)$ there exist smooth solutions $v_{ \pm}(x ; \nu)=v(x ; \nu \pm i 0), v_{ \pm}^{\prime}(x ; \nu)=v^{\prime}(x ; \nu \pm i 0)$ of (2.3), (2.3'). For $\alpha \equiv 0$ and $\alpha=\alpha_{1}+i \alpha_{2}, \alpha_{1}>0, \alpha_{2} \neq 0$, they have the asymptotic behavior as $|x| \rightarrow \infty$ in $R_{+}^{3}$

(2.53) $v(x ; \nu \pm i 0)$

$$
\begin{aligned}
= & 2 \nu g_{ \pm}(x ; \nu) P_{ \pm 1}(\omega)\left[\theta( \pm \omega ; \nu)-C_{ \pm 1}(\tilde{\omega}) \theta( \pm \tilde{\omega} ; \nu)\right] \\
& +O\left(|x|^{-1-\kappa}\right)
\end{aligned}
$$

(2.54) $v^{\prime}(x ; \nu \pm i 0)$

$$
\begin{aligned}
= & 2 \nu g_{ \pm}(x ; \nu) P_{ \pm 1}(\omega)\left[\theta( \pm \omega ; \nu)-C_{ \pm 1}^{\prime}(\tilde{\omega}) \theta( \pm \tilde{\omega} ; \nu)\right] \\
& +O\left(|x|^{-1-\kappa}\right)
\end{aligned}
$$

where

$$
\begin{aligned}
& g_{ \pm}(x ; \nu)=(4 \pi|x|)^{-1} \exp ( \pm i \nu|x| / c), \\
& \theta(\omega ; \nu)=c^{-2} \int_{R_{+}^{3}} \exp (-i \nu \omega y / c) f(y) d y, \\
& \omega=x /|x|, \tilde{\omega}=\left(\omega^{\prime},-\omega_{3}\right),
\end{aligned}
$$

$\kappa \in(0,1)$ is arbitrarily close to $1, P_{ \pm 1}$ are given in $(1.3)$, and $C_{ \pm 1}, C_{ \pm 1}^{\prime}$ are given in (1.7). Modulo $O\left(|x|^{-1-\kappa}\right)$ for positive $t$

$$
\exp (-i \nu t) v_{+}(x, \nu), \quad \exp (i \nu t) v_{-}^{\prime}(x ; \nu)
$$

are outgoing hemispherical waves, while

$$
\exp (-i \nu t) v_{-}(x ; \nu), \quad \exp (i \nu t) v_{+}^{\prime}(x ; \nu)
$$

are incoming hemispherical waves. The order relations are uniform with respect to $x_{3} \geq 0$. For $\alpha=i \alpha_{2}, \alpha_{2} \neq 0, v_{ \pm}(x ; \nu)=v_{R}(x ; \nu \pm i 0)+$ $v_{S}(x ; \nu \pm i 0)$ where $v_{R}(x ; \nu \pm i 0)$ has the asymptotic behavior (2.53). 
For $x_{3} \geq 0,\left|x^{\prime}\right|$ large, and $\nu>0$

(2.57)

$$
\begin{aligned}
& v_{S}(x ; \nu \pm i 0) \\
& \quad=\left|x^{\prime}\right|^{-1 / 2} \exp \left[\nu\left( \pm i\left|x^{\prime}\right|-\tilde{\tau} x_{3}\right) / s\right] S( \pm \gamma) \theta_{S}(\gamma, \nu ; f)+R_{S}^{ \pm}(x ; \nu), \\
& \quad s, S=e, E \text { for } \alpha_{2}>0, s, S=m, M \text { for } \alpha_{2}<0, e=c q>0, \\
& m=-p / \varepsilon>0, \quad \tilde{\tau}_{e}=\mu c q / \alpha_{2}, \quad \tilde{\tau}_{m}=\alpha_{2} / p, \\
& \left|R_{S}^{ \pm}(x ; \nu)\right| \leq \exp \left(-\lambda_{s} x_{3}\right)\left(1+x_{3}\right)\left|x^{\prime}\right|^{-1-\kappa^{\prime}}, \\
& \quad \lambda_{s}=\text { const }>0, \quad \kappa^{\prime} \in(0,1 / 2),
\end{aligned}
$$

while for $\nu<0$

$$
\begin{aligned}
& \text {.58) } \begin{aligned}
v_{S}(x ; \nu & \pm i 0) \\
= & \left|x^{\prime}\right|^{-1 / 2} \exp \left[\nu\left(\mp i|x|-\tilde{\tau}_{s} x_{3}\right) / s\right] S(\mp \gamma) \tilde{\theta}(\gamma, \mu ; f) \\
& \quad+\widetilde{R}^{ \pm}(x ; \nu), \\
s, S= & m, M, \text { for } \alpha_{2}>0, s, S=e, E \text { for } \alpha_{2}<0 .
\end{aligned} \\
& \left|\widetilde{R}_{S}^{ \pm}(x ; \nu)\right| \leq \text { const } \exp \left(-i \tilde{\lambda}_{s} x_{3}\right)\left(1+x_{3}\right)\left|x^{\prime}\right|^{-1-\kappa^{\prime}}, \\
& \tilde{\lambda}_{S}=\text { const }>0, \kappa^{\prime} \in(0,1 / 2) .
\end{aligned}
$$

The exact values of the smooth, scalar functions of $\gamma \in S^{1}, \theta, \tilde{\theta}$ and of the constants $\lambda, \tilde{\lambda}$ can be read from (2.42), (2.47), (2.49), (2.51). The function $(\Lambda-\nu I) v_{S} \in \mathscr{X}$ is orthogonal in $\mathscr{Z}$ to $(\Lambda-\nu I) v_{R} \in$ $\mathscr{X}$. For $\nu, t>0$ modulo $O\left(|x|^{-1-\kappa^{\prime}}\right) \exp (-i \nu t) v_{S}(x ; \nu+i 0)$ is an outgoing cylindrical wave, while $\exp (-i \nu t) v_{S}(x ; \nu-i 0)$ is an incoming cylindrical wave.

3. The uniqueness theorem and the principle of limiting amplitude. In this section we prove uniqueness of solutions of $(2.3),\left(2.3^{\prime}\right)$ in particular radiation classes which contain the solutions constructed in $\S 2$. We then show via the principle of limiting amplitude that the unique solutions of $\S 2$ are the physically interesting solutions.

We define

$$
\begin{aligned}
\mathscr{R}_{ \pm}=\left\{w_{ \pm} \in C^{1}\left(R_{+}^{3} ; C^{6}\right): B w_{ \pm}\left(x,{ }^{\prime}, 0\right)=0,\right. \\
\\
w_{ \pm}(x)=|x|^{-1} \exp ( \pm i \nu|x|) P_{ \pm 1}(\omega) \beta\left(\omega, \nu ; w_{ \pm}\right) \\
+O\left(|x|^{-1-\kappa}\right),|x| \rightarrow \infty, \kappa \in(0,1 / 2), \\
\left.\quad \beta \text { smooth on } S^{2} \text { for each } \nu\right\} .
\end{aligned}
$$

The class $\mathscr{R}_{ \pm}^{\prime}$ is defined in the same way with $B$ replaced by $B^{\prime}$. For 
$\nu>0$ we define the classes of functions $\mathscr{R} S, S=E, M$, as follows: $w_{ \pm} \in R_{ \pm}^{S}$ iff

$$
\begin{aligned}
& \text { (1) } w_{ \pm} \in C^{1}\left(R_{+}^{3}, C^{6}\right), \text { and } w_{ \pm}=w_{ \pm}^{R}+w_{ \pm}^{S}, B W_{ \pm}^{R, S}=0 \text { on } \\
& \left\{x_{3}=0\right\} ; \\
& \text { (2) } W_{ \pm}^{R} \in \mathscr{R}_{ \pm} \text {and for } x_{3} \geq 0,\left|x^{\prime}\right| \text { large } \\
& v_{ \pm}^{S}=\left|x^{\prime}\right|^{-1 / 2} \exp \left[\left(\nu\left( \pm i\left|x^{\prime}\right|-\tilde{\tau}_{s} x_{3}\right) / s\right)\right] S( \pm \gamma) \theta_{s}(\gamma ; \nu) \\
& \quad+R_{s}^{ \pm}(x ; \nu) \text {. } \\
& \left|R_{s}^{ \pm}(x ; \nu)\right| \leq \text { const }\left|x^{\prime}\right|^{-1-\kappa^{\prime}} h\left(x_{3}\right), \quad h \in L_{2}\left(R_{+}\right), \kappa^{\prime} \in(0,1 / 2) ; \\
& \text { (3) }(\Lambda-\nu I) w_{ \pm}^{R} \in \mathscr{X} \text { is orthogonal to }(\Lambda-\nu I) v_{ \pm}^{S} \in \mathscr{X} ; \\
& \text { (4) for each } 0<R<\infty \\
& \lim _{L \rightarrow \infty} \int_{\left|x^{\prime}\right| \leq R} d x^{\prime} \bar{t}_{v} v_{ \pm}^{S}\left(x^{\prime}, L\right) A_{3} v_{ \pm}^{S}(x ; L) \rightarrow 0, \\
& \text { and }{ }^{t} S( \pm \gamma) A(\gamma, 0) S( \pm \gamma)=\text { const } \neq 0, \gamma \in S^{1} .
\end{aligned}
$$

For $\nu<0$ the class $\mathscr{R}_{ \pm}^{S} \omega$ is defined in the same way except that in (2) $\pm i\left|x^{\prime}\right| \rightarrow \mp i\left|x^{\prime}\right|$ and $S( \pm \gamma) \rightarrow S(\mp \gamma)$.

Remark. Condition (4) for $v_{S}(x ; \nu \pm i 0), S=E, M$, in the present case is automatically satisfied, since

$$
{ }^{\bar{t}} v_{S}\left(x^{\prime}, L ; \nu \pm i 0\right) A_{3} v_{S}\left(x^{\prime}, L ; \nu \pm i 0\right)=0
$$

for any $L>0$ (this follows from (2.31) and the ensuing argument, since $\left.{ }^{\bar{t}} S(\xi) A_{3} S(\xi)=0, S=E, M\right)$. In more general situations, however, (e.g., elasticity) (4) is satisfied but not trivially as here. The second part of condition (4) is satisfied because of (2.43), (2.48). Otherwise, the fact that the $v(x ; \nu \pm i 0)$ constructed in $\S 2$ belong to $\mathscr{R}_{ \pm}$ or $\mathscr{R} \pm$ follows from Theorem 2.1 .

THEOREM 3.1. Suppose $w_{ \pm}, w_{ \pm}^{\prime}$ are solutions of $(2.3),\left(2.3^{\prime}\right)$ with $f \in C_{0}^{\infty}\left(R_{+}^{3}, C^{6}\right)$ and $0 \neq \nu \in R$. In the nonselfadjoint case $w_{+}$is unique in $\mathscr{R}_{+}$and $w_{-}^{\prime}$ is unique in $\mathscr{R}_{-}$(these are the outgoing solutions). In the selfadjoint case $\alpha \equiv 0 w_{ \pm}$are unique in $\mathscr{R}_{ \pm}$. In the selfadjoint case $\alpha=i \alpha_{2}, \alpha_{2} \neq 0, w_{ \pm}$are unique in $\mathscr{R} S$.

COROLlaRY. If $\alpha \equiv 0$ or $\alpha=i \alpha_{2}, \alpha_{2} \neq 0$, the solutions $v_{ \pm}(x ; \nu)$ of (2.3) constructed in $\S 2$ are unique in $\mathscr{R}_{ \pm}$or $\mathscr{R}_{ \pm}^{S}$ respectively. In the case $\alpha_{1}>0 v_{+}(x ; \nu)$ is unique in $\mathscr{R}_{+}$, and $v_{-}^{\prime}(x ; \nu)$ is unique in $\mathscr{R}_{-}^{\prime}$. 
Proof of Theorem 3.1. We first consider the cases $\alpha \equiv 0$ or $\alpha=$ $\alpha_{1}+i \alpha_{2}, \alpha_{1}>0$. Thus, suppose $w, w^{\prime} \in C^{1}\left(R^{3}, C^{6}\right)$ and for $\nu \neq 0$ $(\Lambda-\nu I) w=0,(\Lambda-\nu I) w^{\prime}=0$ in $R_{+}^{3}, B w\left(x^{\prime}, 0\right)=0, B^{\prime} w^{\prime}\left(x^{\prime}, 0\right)=0$. Integration by parts in the intersection of $R_{+}^{3}$ with a ball of radius $R$ then gives for $u=w, w^{\prime}$

$$
\begin{aligned}
0= & (u,[\Lambda-\nu I])_{B_{R}}-([\Lambda-\nu I] u, u)_{B_{R}} \\
= & i \int_{\left|x^{\prime}\right|<R}{ }^{\bar{t}} u\left(x^{\prime}, 0\right) A_{3} u\left(x^{\prime}, 0\right) d x^{\prime} \\
& -i \int_{R_{+}^{3} \cap\{|x|=R\}}{ }^{\bar{t}} u(x) A(\omega) u(x) d S_{R}(x)
\end{aligned}
$$

where $A_{3}$ is the coefficient matrix of $D_{3}$ in $(0.1)$ and $\omega=x /|x|$. Since $B w\left(x^{\prime}, 0\right)=0, B^{\prime} w^{\prime}\left(x^{\prime}, 0\right)=0$ and $A_{3} u={ }^{t}\left(u_{5},-u_{4}, 0,-u_{2}, u_{1}, 0\right)$, we thus have

$$
\begin{aligned}
& \int_{R_{+}^{3} \cap\{|x|=R\}}{ }^{\bar{t}} w(x) A(\omega) w(x) d S_{R}(x) \\
& =-2 \alpha_{1} \int_{\left|x^{\prime}\right|<R}\left[\left|w_{4}\left(x^{\prime}, 0\right)\right|^{2}+\mid w_{5}\left(x^{\prime},\left.0\right|^{2}\right] \leq 0\right.
\end{aligned}
$$

$$
\begin{aligned}
& \int_{R_{+}^{3} \cap\{|x|=R\}}{ }^{\bar{t}} w^{\prime}(x) A(\omega) w^{\prime}(x) d S_{R}(x) \\
& \quad=2 \alpha_{1} \int_{\left|x^{\prime}\right| \leq R}\left[\left|w_{4}^{\prime}\left(x^{\prime}, 0\right)\right|+\left|w_{5}^{\prime}\left(x^{\prime}, 0\right)\right|^{2}\right] \geq 0 .
\end{aligned}
$$

If now $w=w_{ \pm} \in \mathscr{R}_{ \pm}, w^{\prime}=w_{ \pm}^{\prime} \in \mathscr{R}_{ \pm}^{\prime}$, then from (3.1), (3.3), (3.3') and the fact that $A(\omega) P_{ \pm 1}(\omega)= \pm P_{ \pm 1}(\omega)$, we have

$$
\begin{array}{r} 
\pm \int_{S^{2}}{ }^{\bar{t}}\left[P_{ \pm 1}(\omega) \beta\left(\omega, \nu ; w_{ \pm}\right)\right] P_{ \pm 1}(\omega) \beta\left(\omega, \nu ; w_{ \pm}\right) d S+O\left(R^{-\kappa}\right) \\
=-2 \alpha_{1} \int_{\left|x^{\prime}\right| \leq R}\left[\left|w_{4}\left(x^{\prime}, 0\right)\right|^{2}+\left|w_{5}\left(x^{\prime}, 0\right)\right|^{2}\right] d x^{\prime} \leq 0
\end{array}
$$

$$
\begin{gathered}
\pm \int_{S^{2}}{ }^{\bar{t}}\left[P_{ \pm 1}(\omega) \beta\left(\omega, \nu ; w_{ \pm}^{\prime}\right)\right] P_{ \pm 1}(\omega) \beta\left(\omega, \nu ; w_{ \pm}^{\prime}\right) d S+O\left(R^{-\kappa}\right) \\
\quad=2 \alpha_{1} \int_{\left|x^{\prime}\right| \leq R}\left[\left|w_{4}^{\prime}\left(x^{\prime}, 0\right)\right|^{2}+\left|w_{5}^{\prime}\left(x^{\prime}, 0\right)\right|^{2}\right] d x^{\prime} \geq 0 .
\end{gathered}
$$

Letting $R \rightarrow \infty$, it follows from (3.4) in the selfadjoint case $\alpha \equiv 0$ that $w_{ \pm}=O\left(|x|^{-1-\kappa}\right)$, whence, since $\kappa>1 / 2, w_{ \pm} \in \mathscr{X}$ and is thus 
an eigenfunction. Now zero is the only eigenvalue and $\nu \neq 0$, so that $w_{ \pm} \equiv 0$. The assertions for $w_{+}$and $w_{-}^{\prime}$ in the nonselfadjoint case follow in the same way.

We now suppose that $w_{ \pm} \in \mathscr{R}_{ \pm}^{S}$ and $(\Lambda-\nu I) w_{ \pm}(x)=0$ in $R_{ \pm}^{3}, \nu \neq 0$, $w_{ \pm}=w_{ \pm}^{R}+w_{ \pm}^{S}$. Condition (3) of (3.2) implies that $(\Lambda-\nu I) w_{ \pm}^{R}=0$ and $(\Lambda-\nu I) w_{ \pm}^{S}(x)=0$ individually. It follows just as above that $W_{ \pm}^{R} \in \mathscr{X}$. Now let $C_{R}^{L}=\left\{x \in R_{ \pm}^{3}:\left|x^{\prime}\right| \leq R, 0 \leq x_{3} \leq L\right\}$. Then, since $B w_{ \pm}^{S}\left(x^{\prime}, 0\right)=0$ implies ${ }^{\bar{t}} w_{ \pm}^{S}\left(x^{\prime}, 0\right) A_{3} w_{ \pm}^{S}\left(x^{\prime}, 0\right)=0$, we have for $\gamma \in S^{1}$

$$
\begin{aligned}
0= & \left(w_{ \pm}^{S},[\Lambda-\nu I] w_{ \pm}^{S}\right)_{C_{R}^{L}}-\left([\Lambda-\nu I] w_{ \pm}^{S}, w_{ \pm}^{S}\right)_{C^{L}} \\
& -i \int_{\left|x^{\prime}\right| \leq R}{ }^{\bar{t}} w_{ \pm}^{S}\left(x^{\prime}, L\right) A_{3} w_{ \pm}^{S}\left(x^{\prime}, L\right) \\
& -i \int_{0}^{L} \int_{\left|x^{\prime}\right|=R}{ }^{\bar{t}} w_{ \pm}^{S}(x) A(\gamma, 0) w_{ \pm}^{S}(x) d x^{\prime} d x_{3} .
\end{aligned}
$$

Letting $L \rightarrow \infty$ and using condition (4) of (3.2), it follows that

$$
0=\int_{0}^{\infty} \int_{\left|x^{\prime}\right|=R}{ }^{\bar{t}} w_{ \pm}^{S}(x) A(\gamma, 0) w_{ \pm}^{S}(x) d x^{\prime} d x_{3}
$$

Now from condition (2) of (3.2) we have

$$
\begin{aligned}
0= & \int_{S^{1}} \int_{0}^{\infty} \exp \left(-2 \tau x_{3} / s\right)\left|\theta_{S}(x ; \nu)\right|^{2 \bar{t}} S( \pm \gamma) \\
& \times A(\gamma, 0) S( \pm \gamma, 0) d x_{3} d \phi \\
& +\int_{0}^{\infty} d x_{3} \int_{\left|x^{\prime}\right|=R} \exp \left[\left(\mp i\left|x^{\prime}\right|-\tau_{S} x_{3}\right) / s\right] \bar{\theta}(\gamma ; \nu){ }^{\bar{t}} S( \pm \gamma) \\
& \times A(\gamma, 0) R_{S}^{ \pm}(x ; \nu) R^{1 / 2} d \phi \\
& +\int_{0}^{\infty} d x_{3} \int_{\left|x^{\prime}\right|=R} \exp \left[\left( \pm i\left|x^{\prime}\right|-\tau_{S} x_{3}\right) / s\right]{ }^{\bar{t}} R_{S}^{ \pm}(x ; \nu) \theta_{S}(\gamma ; \nu) \\
& \times A(\gamma, 0) S( \pm \gamma) R^{1 / 2} d \phi \\
& +\int_{0}^{\infty} d x_{3} \int_{\left|x^{\prime}\right|=R}{ }^{\bar{t}} R_{S}^{ \pm}(x ; \nu) A(\gamma, 0) R_{S}^{ \pm}(x ; \nu) R d \phi .
\end{aligned}
$$

From (3.2) and Cauchy's inequality the second and third terms are bounded by const $R^{-1 / 2-\kappa^{\prime}}|h|_{L_{2}(R)_{+}}^{2}$. The last term is bounded by const $R^{-1-2 \kappa^{\prime}}|h|_{L_{2}(R)_{+}}^{2}$. Thus, letting $R \rightarrow \infty$ in (3.5) and integrating on $x_{3}$, 
we have

$$
0=\int_{S^{1}}\left|\theta_{S}(\gamma ; \nu)\right|^{2 \bar{t}} S( \pm \gamma) A(\gamma, 0) S( \pm \gamma) d \phi
$$

From the second part of condition (4) it now follows that $\theta_{S}(\gamma ; \nu)=0$ for a.e. $\gamma \in S^{1}$. Since $R_{S}^{ \pm}(x ; \nu) \in \mathscr{X}$, we thus find that also $w_{S}^{ \pm} \in \mathscr{X}$. Hence, $w_{ \pm}=w_{ \pm}^{R}+w_{ \pm}^{S} \in \mathscr{X}$, so, as above, $w \equiv 0$. This completes the proof of Theorem 3.1.

Because of the simple structure of the spectrum, in $\S 2$ we were able to construct solutions of $(2.3),\left(2.3^{\prime}\right)$ from both sides of the real axis even in the nonselfadjoint case. Our uniqueness proof, however, works only for solutions obtained from the side of the real axis in the resolvent set. There is some obscure justice in this: the outgoing solutions $w_{+}, w_{-}^{\prime}$ are the physically interesting solutions, since timeharmonic incoming solutions are obtained via limiting amplitude by going backward in time which is impossible in the nonselfadjoint case. We proceed to demonstrate all this.

Let $u(x, t), u_{3}^{\prime}(x, t)$ be the solutions in $\mathscr{X}$ of problems $(0.2),(0.3)$ with $f \in C_{0}^{\infty}\left(R_{+}^{3}, C^{6}\right)$ with $u(x, 0)=0, u^{\prime}(x, 0)=0$. Then by Duhamel's principle we have

$$
\begin{aligned}
& u(x, t)=i \int_{0}^{t}[S(t-\tau) f](x) \exp (-i \nu \tau) d \tau \\
& u^{\prime}(x, t)=-i \int_{0}^{t}\left[S^{*}(t-\tau) f\right](x) \exp (i \nu \tau) d \tau
\end{aligned}
$$

By a change of variables we have from (1.14)

$$
\begin{aligned}
& \exp (i \nu t) u(x, t)=i \int_{0}^{t} \exp (i \nu \tau)[S(t) f](x) d \tau \\
& =i \int_{0}^{t} \exp (i \nu \tau)\left[\Pi_{0} f(x)+\Psi_{1}^{*} \exp (-i c|\cdot| \tau) \Psi_{1}^{\prime} f(x)\right. \\
& +\Psi_{-1}^{*} \exp (i c|\cdot| \tau) \Psi_{-1}^{\prime} f(x) \\
& +\Sigma_{E}^{*} \exp (-i e(\cdot) \tau) \Sigma_{E}^{\prime} f(x) \\
& \left.\quad+\Sigma_{M}^{*} \exp (-i m(\cdot) \tau) \Sigma_{M}^{\prime} f(x)\right] d \tau \\
& \equiv w_{0}(x, t)+w_{1}(x, t)+w_{-1}(x, t)+w_{E}(x \cdot t)+w_{M}(x \cdot t)
\end{aligned}
$$




$$
\begin{aligned}
& \left(3.7^{\prime}\right) \exp (-i \nu t) u^{\prime}(x, t)=-i \int_{0}^{t} \exp (-i \nu \tau)\left[S^{*}(\tau) f\right](x) d \tau \\
& =-i \int_{0}^{t} \exp (-i \nu \tau)\left[\Pi_{0} f(x)+\left(\Psi_{1}^{\prime}\right)^{*} \exp (i c|\cdot| \tau) \Psi_{1} f(x)\right. \\
& +\left(\Psi_{-1}^{\prime}\right)^{*} \exp (-i c|\cdot| \tau) \Psi_{-1} f(x) \\
& +\left(\Sigma_{E}^{\prime}\right)^{*}(i \bar{e}(\cdot) \tau) \Sigma_{E} f(x) \\
& \left.+\left(\Sigma_{M}^{\prime}\right)^{*} \exp (i \bar{m}(\cdot) \tau) \Sigma_{M} f(x)\right] d \tau \\
& \equiv w_{0}^{\prime}(x, t)+w_{1}^{\prime}(x, t)+w_{-1}^{\prime}(x, t)+w_{E}^{\prime}(x, t)+w_{M}^{\prime}(x \cdot t) .
\end{aligned}
$$

It is clear that the limit as $t \rightarrow+\infty$ of $w_{0}(x, t)$ does not exist unless $\Pi_{0} f(x)=0$ which we henceforth assume. As in (2.9), (2.10) with integration by parts

$$
\begin{aligned}
&-c^{2} \tau^{2} \Psi_{1}^{*} \exp (-i c|\cdot| \tau) \Psi_{1}^{\prime} f(x) \\
&=(2 \pi)^{-3 / 2} \int_{S^{2}} d S \int_{0}^{\infty} \exp (i c|\eta| \tau) \partial_{|\eta|}^{2} \\
& \quad \times\left[\exp (i x \eta) \check{f}_{1}(\eta)|\eta|^{2}\right] d|\eta|
\end{aligned}
$$

has finite modulus, so there exists $(\nu>0)$

(3.8) $\lim _{t \rightarrow \infty} w_{1}(x, t)$

$$
\begin{aligned}
& =i \int_{0}^{\infty} \exp (i \nu \tau) \Psi_{1}^{*} \exp (-i c|\cdot| \tau) \Psi_{1}^{\prime} f(x) d \tau \\
& =i(2 \pi)^{-3 / 2} \int_{0}^{\infty} \int_{R^{3}} \exp [i \tau(\nu-c|\eta|)] \exp (i x \eta) \check{f}_{1}(\eta) d(\eta) d \tau \\
& =i(2 \pi)^{-3 / 2} \lim _{\varepsilon \rightarrow 0} \int_{0}^{\infty} \exp (-\varepsilon \tau) d \tau \int_{R^{3}} \exp [i \tau(\nu-c|\eta|)] \\
& \quad \times \exp (i x \eta) \check{f}_{1}(\eta) d \eta \\
& =(2 \pi)^{-3 / 2} \lim _{\varepsilon \rightarrow 0} \int_{R^{3}} \exp (i x \eta)[c|\eta|-(\nu+i \varepsilon)]^{-1} \check{f}_{1}(\eta) d \eta \\
& =v_{1}(x ; \nu+i 0)
\end{aligned}
$$

of part $2, \S 2$. Continuing in this manner, we see that there exists the limit as $t \rightarrow+\infty$ of $\exp (i \nu t) u(x, t)=v(x ; \nu+i 0)$ where $v(x ; \nu+i 0)$ is the solution of Theorem 2.1.

In exactly the same way as in (3.6) there exists

$$
\begin{aligned}
\lim _{t \rightarrow \infty} & -i \int_{0}^{t}\left[\exp (-i \nu \tau)\left(\Psi_{1}^{\prime}\right)^{*} \exp (i c|\cdot| \tau) \Psi_{1} f\right](x) d \tau \\
& =v_{1}^{\prime}(x ; \nu-i 0)
\end{aligned}
$$


of part $4, \S 2$, and, continuing in this manner, we conclude from $\left(3.7^{\prime}\right)$ that there exists the limit as $t \rightarrow \infty$ of $\exp (-i \nu t) u^{\prime}(x, t)$ which is equal to $v^{\prime}(x ; \nu-i 0)$, the solution of Theorem 2.1 .

Further, in the selfadjoint cases

$$
\begin{aligned}
\lim _{t \rightarrow-\infty} & w_{1}(x, t) \\
\quad= & -i(2 \pi)^{-3 / 2} \int_{-\infty}^{0} \int_{R^{3}} \exp [i \tau(\nu-c|\eta|)] \exp (i x \eta) \check{f}_{1}(\eta) d \eta d \tau \\
\quad= & \lim _{\varepsilon \rightarrow 0} \int_{R^{3}}[c|\eta|-(\nu-i \varepsilon)]^{-1} \exp (i x \eta) \check{f}_{1}(\eta) d \eta \\
= & v_{1}(x ; \nu-i 0)
\end{aligned}
$$

of part 2, $\S 2$, and in this way from (3.7) we see that there exists the limit as $t \rightarrow-\infty$ of $\exp (i \nu t) u(x, t)$ which is equal to $v(x ; \nu-i 0)$ where the latter is the solution of Theorem 2.1. We summarize these results as follows.

THEOREM 3.2. Let $f \in C_{0}^{\infty}\left(R_{+}^{3}, C^{6}\right)$ be in the complement of the null space of $\Lambda$, and let $u(x, t), u^{\prime}(x, t)$ be the solutions in $\mathscr{X}$ of $(0.2),(0.3)$ with $u(x, 0)=0, u^{\prime}(x, 0)=0$. Then in the selfadjoint case

$$
\lim _{t \rightarrow \pm \infty} \exp (i \nu t) u(x, t)=v(x ; \nu \pm i 0)
$$

where $v(x ; \nu \pm i 0)$ are the solutions of $(2.3)$ considered in Theorem 2.1. In the nonselfadjoint cases

$$
\begin{aligned}
& \lim _{t \rightarrow \infty} \exp (i \nu t) u(x, t)=v(x ; \nu+i 0), \\
& \lim _{t \rightarrow \infty} \exp (-i \nu t) u^{\prime}(x, t)=v^{\prime}(x ; \nu-i 0),
\end{aligned}
$$

where the right sides are the outgoing solutions of (2.3), (2.3)' of Theorem 2.1 .

Concluding remarks. In the applied literature it is often assumed that the source is a time-harmonic point source, e.g., an oscillating dipole. The spatial part of the response is then sought as a solution of the Helmholtz equation (a Hertz potential), and a prescription is given for constructing the electric and magnetic fields from this potential. What this really amounts to is a specialization of the Green function for the original problem for Maxwell's equations. In the present case the Green functions can be read off from the material above (as the kernel of $\mathscr{G}_{ \pm}$in the expression $v_{ \pm}=\mathscr{G}_{ \pm} f$ ), but a simple expression (without transforms) is obtained only in the case $\alpha=0$, i.e., the case of the classical boundary condition. It seems worthwhile to present this expression. 
For Maxwell's equations in all of $R^{3}$ the outgoing $(+)$ and incoming (-) Green functions for the time dependence $\exp (-i \nu t)$ are $(\varepsilon=$ $\mu=1)$

$$
G_{ \pm}(x, y ; \nu)=\left(\nu I+A\left(D_{x}\right)+\nu^{-1}\left[\begin{array}{cc}
\partial_{x} \otimes \partial_{x} & 0 \\
0 & \partial_{x} \otimes \partial_{x}
\end{array}\right]\right) g_{ \pm}(x-y ; \nu),
$$

where $g_{ \pm}(x ; \nu)$ are the functions of $(2.32)$, the matrix $[\partial \otimes \partial]_{i j}=\partial_{i} \partial_{j}$, and on functions $f={ }^{t}\left(f^{1}, f^{2}\right) \in C_{0}^{\infty}\left(R^{3}, C^{6}\right)$ in $\mathscr{D}^{\prime}$

$$
\begin{aligned}
\operatorname{rot}_{x} g_{ \pm}(x-; \nu)\left(f^{i}\right) & =g_{ \pm}(x-; \nu)\left(\operatorname{rot} f^{i}\right), \\
\partial_{x} \otimes \partial_{x} g_{ \pm}(x-; \nu)\left(f^{i}\right) & =g_{ \pm}(x-; \nu)\left(\partial \otimes \partial f^{i}\right), \quad i=1,2 .
\end{aligned}
$$

It is straightforward to verify that

$$
G_{+}(x, y ; \nu)\left(\begin{array}{c}
\nu a \\
0
\end{array}\right)=\left(\begin{array}{c}
\nu^{2} g_{+}(x-y ; \nu) a+\partial \otimes \partial g_{+}(x-y ; \nu) a \\
-i \nu \operatorname{rot} g_{+}(x-y ; \nu) a
\end{array}\right)
$$

is the usual response to an electric dipole at the point $y \in R^{3}$ with direction $a \in R^{3}$.

For $R_{+}^{3}$ and the classical boundary condition $(\alpha=0)$ the Green functions are

$$
\mathscr{G}_{ \pm}(x, y ; \nu)=G_{ \pm}(x, y ; \nu)-R_{ \pm}(x, y ; \nu)
$$

$$
\begin{aligned}
& R_{ \pm}(x, y ; \nu) \\
& =\left(\nu I+A\left(D_{x}\right)+\nu^{-1}\left[\begin{array}{cc}
\partial_{x} \otimes \partial_{x} & 0 \\
0 & \partial_{x} \otimes \partial_{x}
\end{array}\right]\right) Q g_{ \pm}(x,-\tilde{y} ; \nu), \\
& \tilde{y}=\left(y^{\prime},-y_{3}\right)
\end{aligned}
$$

where $Q$ is the matrix of (1.7).

We leave to the reader as an interesting exercise the verification that $\mathscr{G}_{ \pm}(x, \cdot ; \nu)(f)$ has the asymptotic behavior $(2.53)$ and that the response with the classical boundary condition to the electric dipole above is

$$
\mathscr{G}_{+}(x, y ; \nu)\left(\begin{array}{c}
\nu a \\
0
\end{array}\right) \text {. }
$$

\section{REFERENCES}

[1] D. Eidus, The limiting absorption principle for the diffraction problem with two unbounded media, Comm. Math. Phys., 107 (1986), 29-39.

[2] D. S. Gilliam and J. R. Schulenberger, Electromagnetic waves in a three-dimensional conducting half space, J. Math. Anal. Appl., 89, No. 1, (1982), 129-185.

[3] __, On the structure of surface waves, Adv. in Appl. Math., 4 (1983), 212-243. 
[4] M. Matsumura, Comportement des solutions de quelques problèmes mixtes pour certains systèmes hyperboliques symétriques à coefficients constants, Publ. RIMS Kyoto, Ser. A, 4, No. 2, (1968), 309-359.

[5] _ Asymptotic behavior at infinity for Green functions of first order systems with characteristics of nonuniform multiplicity, Publ, RIMS Kyoto, 12, No. 2, (1976), 317-377.

[6] S. G. Mikhlin, Multidimensional singular integrals and integral equations, Oxford, Pergamon Press (1965).

[7] J. R. Schulenberger and C. H. Wilcox, A Rellich uniqueness theorem for steadystate wave propagation in inhomogeneous, anisotropic media, Arch. Rat. Mech. Anal., 41, No. 1, (1971), 18-45.

[8] J. R. Schulenberger, Boundary waves on perfect conductors, J. Math. Anal. Appl., 66, No. 3, (1978), 514-549.

[9] B. R. Vainberg, Asymptotic methods in equations of mathematical physics, Izd. Mosk. Univ., (1982).

[10] S. Wakabayashi, The principle of limiting amplitude for symmetric hyperbolic systems of first order in the half space $R_{+}^{n}$, Publ. RIMS Kyoto, 11, (1975), 149162.

Received April 27, 1988.

427 E. NORTON

TUCSON, AZ 85719 



\section{PACIFIC JOURNAL OF MATHEMATICS EDITORS}

\author{
V. S. VARADARAJAN \\ (Managing Editor) \\ University of California \\ Los Angeles, CA 90024-1555-05 \\ Herbert Clemens \\ University of Utah \\ Salt Lake City, UT 84112 \\ ThOMAs EnRIGHT \\ University of California, San Diego \\ La Jolla, CA 92093
}

R. FINN

Stanford University

Stanford, CA 94305

HeRmanN FlaschKa

University of Arizona

Tucson, AZ 85721

VAUGHAN F. R. Jones

University of California

Berkeley, CA 94720

SteVEn KerckHofF

Stanford University

Stanford, CA 94305

\author{
ROBION KIRBY \\ University of California \\ Berkeley, CA 94720 \\ C. C. MOORE \\ University of California \\ Berkeley, CA 94720 \\ HAROLd StaRK \\ University of California, San Diego \\ La Jolla, CA 92093
}

\section{ASSOCIATE EDITORS}
R. ARENS
E. F. BECKENBACH
B. H. NeUMANN
F. WoLF
K. YoshidA (1906-1982)
(1904-1989)

\section{SUPPORTING INSTITUTIONS}

\section{UNIVERSITY OF ARIZONA UNIVERSITY OF BRITISH COLUMBIA CALIFORNIA INSTITUTE OF TECHNOLOGY UNIVERSITY OF CALIFORNIA MONTANA STATE UNIVERSITY UNIVERSITY OF NEVADA, RENO NEW MEXICO STATE UNIVERSITY OREGON STATE UNIVERSITY}

\author{
UNIVERSITY OF OREGON \\ UNIVERSITY OF SOUTHERN CALIFORNIA \\ STANFORD UNIVERSITY \\ UNIVERSITY OF HAWAII \\ UNIVERSITY OF TOKYO \\ UNIVERSITY OF UTAH \\ WASHINGTON STATE UNIVERSITY \\ UNIVERSITY OF WASHINGTON
}

The Supporting Institutions listed above contribute to the cost of publication of this Journal, but they are not owners or publishers and have no responsibility for its content or policies.

Mathematical papers intended for publication in the Pacific Journal of Mathematics should be in typed form or offset-reproduced (not dittoed), double spaced with large margins. Please do not use built up fractions in the text of the manuscript. However, you may use them in the displayed equations. Underline Greek letters in red, German in green, and script in blue. The first paragraph must be capable of being used separately as a synopsis of the entire paper. In particular it should contain no bibliographic references. Please propose a heading for the odd numbered pages of less than 35 characters. Manuscripts, in triplicate, may be sent to any one of the editors. Please classify according to the scheme of Math. Reviews, Index to Vol. 39. Supply name and address of author to whom proofs should be sent. All other communications should be addressed to the managing editor, or Elaine Barth, University of California, Los Angeles, California 90024-1555-05.

There are page-charges associated with articles appearing in the Pacific Journal of Mathematics. These charges are expected to be paid by the author's University, Government Agency or Company. If the author or authors do not have access to such Institutional support these charges are waived. Single authors will receive 50 free reprints; joint authors will receive a total of 100 free reprints. Additional copies may be obtained at cost in multiples of 50 .

The Pacific Journal of Mathematics is issued monthly as of January 1966. Regular subscription rate: $\$ 190.00$ a year (6 Vols., 12 issues). Special rate: $\$ 95.00$ a year to individual members of supporting institutions.

Subscriptions, orders for numbers issued in the last three calendar years, and changes of address should be sent to Pacific Journal of Mathematics, P.O. Box 969, Carmel Valley, CA 93924, U.S.A. Old back numbers obtainable from Kraus Periodicals Co., Route 100, Millwood, NY 10546.

The Pacific Journal of Mathematics at P.O. Box 969, Carmel Valley, CA 93924 (ISSN 0030-8730) publishes 6 volumes per year. Second-class postage paid at Carmel Valley, California 93924, and additional mailing offices. Postmaster: send address changes to Pacific Journal of Mathematics, P.O. Box 969, Carmel Valley, CA 93924.

\section{PUBLISHED BY PACIFIC JOURNAL OF MATHEMATICS, A NON-PROFIT CORPORATION} Copyright (C) 1990 by Pacific Journal of Mathematics 


\section{Pacific Journal of Mathematics}

Vol. 142, No. $2 \quad$ February, 1990

Christopher J. Bishop, Bounded functions in the little Bloch space . . . . . 209

Lutz Bungart, Piecewise smooth approximations to $q$-plurisubharmonic

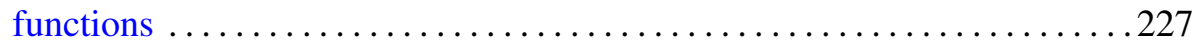

Donald John Charles Bures and Hong Sheng Yin, Outer conjugacy of

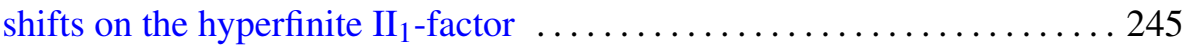

A. D. Raza Choudary, On the resultant hypersurface $\ldots \ldots \ldots \ldots \ldots 259$

Luis A. Cordero and Robert Wolak, Examples of foliations with foliated

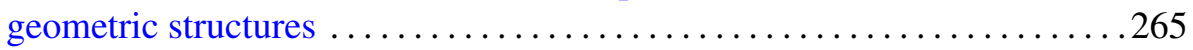

Peter J. Holden, Extension theorems for functions of vanishing mean

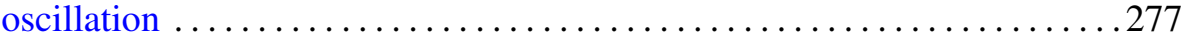

Detlef Müller, A geometric bound for maximal functions associated to convex bodies ........................................ 297

John R. Schulenberger, Time-harmonic solutions of some dissipative problems for Maxwell's equations in a three-dimensional half space . . . 313

Mark Andrew Smith and Barry Turett, Normal structure in Bochner

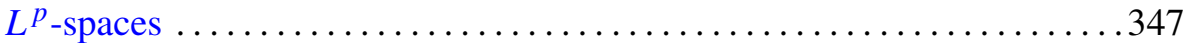

Jun-ichi Tanaka, Blaschke cocycles and generators ................... 357

R. Z. Yeh, Hyperholomorphic functions and higher order partial differential equations in the plane ................................ 379 